\begin{tabular}{|c|c|}
\hline 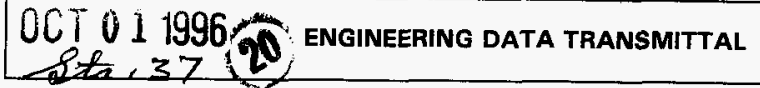 & 1. ЕDT 618229 \\
\hline
\end{tabular}

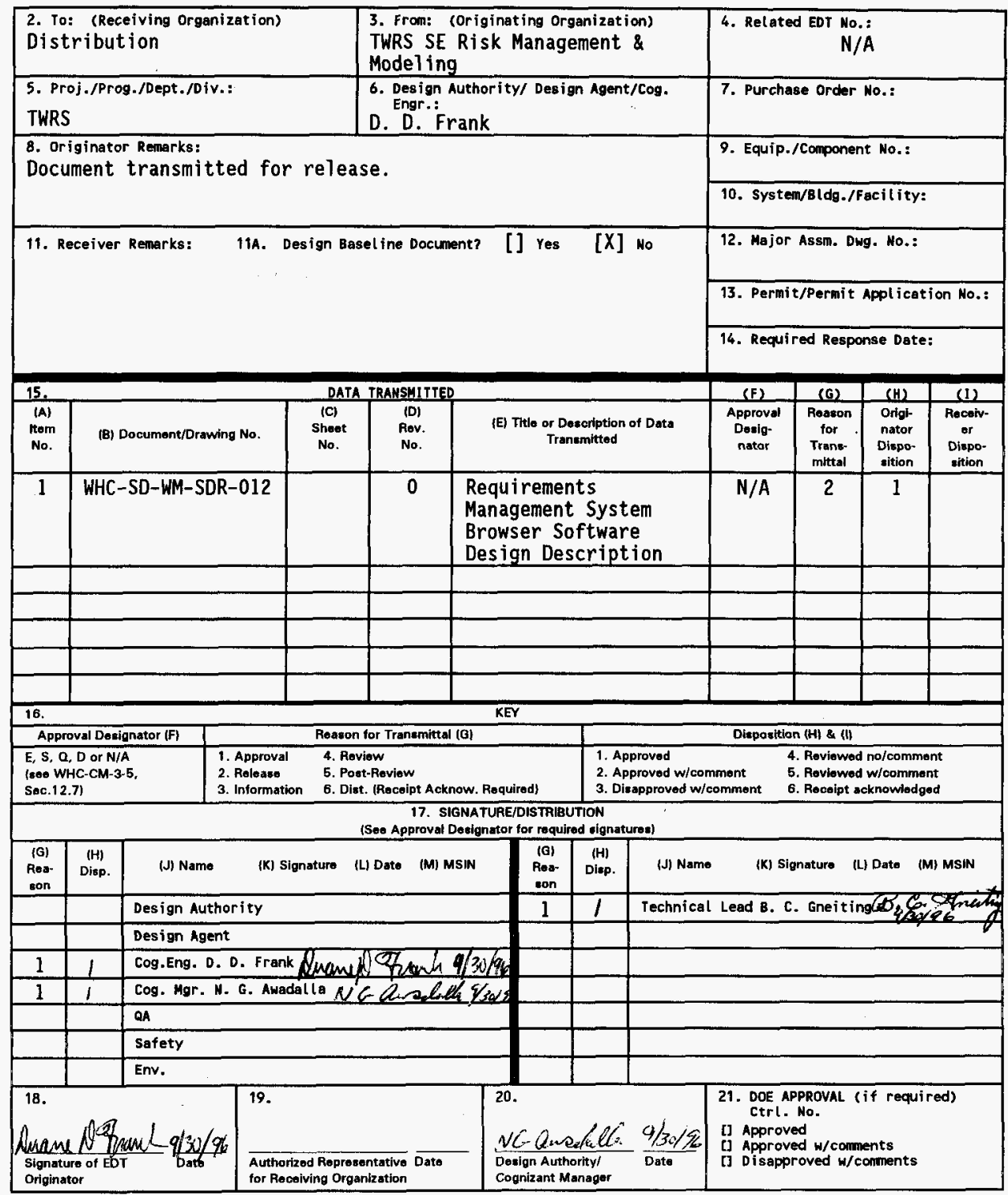

BD-7400-172-2 (05/96) GEF097 


\section{Requirements Management System Browser Software Design Description}

\section{D. Frank}

Westinghouse Hanford Company, Richland, WA 99352

U.S. Department of Energy Contract DE-AC06-87RL10930

\begin{tabular}{|c|c|c|}
\hline $\begin{array}{l}\text { EDT/ECN: } \\
\text { Org Code: } \\
\text { B\&R Code: }\end{array}$ & $\begin{array}{l}618229 \\
74400 \\
E W 3120075\end{array}$ & $\begin{array}{l}\text { UC: } 905 \\
\text { Charge Code: } \\
\text { Total Pages: }\end{array}$ \\
\hline
\end{tabular}

Key Words: $\quad$ Systems Engineering; Requirements Management; RMACs; TWRS; Browser; Parser; SE Tools

Abstract: The purpose of this document is to provide an "as-built" design description for the Requi rements Management Systen Browser (RMSB) application. The Graphical User Interface (GUI) and database structure design are described for the RMSB application, referred to as the "Browser." The RMSB application provides an easy to use $P C$-based interface to browse systems engineering data stored and managed in a UNIX software application. The system engineering data include functions, requirements, and architectures that make up the Iank Waste Remediation System (TWRS) technical basel ine.

Trademarks: Access, Visual Basic, Windows, and SQL are trademarks of Microsoft Corporation, Redmond, WA.

Unix is a trademark of IBM Corporation.

TRADEMARK DISCLAIMER. Reference herein to any specific commercial product, process, or service by trade name, trademark, manufacturer, or otherwise, does not necessarily constitute or imply its endorsement, recommendation, or favoring by the United States Government or any agency thereof or its contractors or subcontractors.

Printed in the United States of America. To obtain copies of this document, contact: WHC/BCS Dociment Control Services, P.O. Box 1970, Mailstop H6-08, Richland WA 99352, Phone (509) 372-2420; Fax (509) 376-4989.
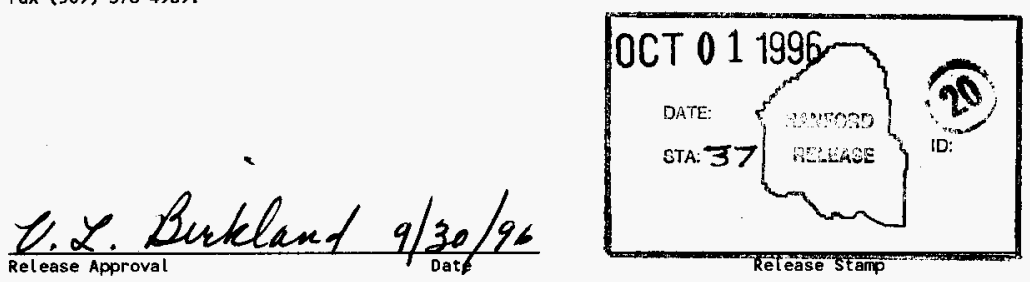

\section{Approved for Public Release}




\section{CONTENTS}

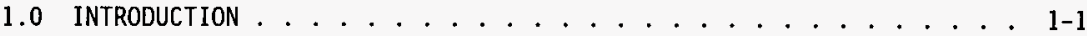

2.0 PURPOSE AND SCOPE ..................... 2-1

3.0 ABBREVIATIONS AND ACRONYMS ................... 3-1

4.0 DESIGN DESCRIPTION . . . . . . . . . . . . . . . 4-1

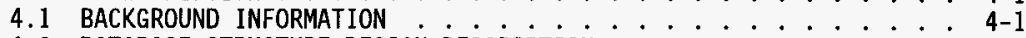

4.2 DATABASE STRUCTURE DESIGN DESCRIPTION .......... . . 4-2

4.2.1 Element Tables ................ . . 4-2

4.2.2 Relation Tables ............... . . 4-6

4.2.3 Change Tables .............. . . . 4-7

4.3 FORM (WINDOW) DESIGN DESCRIPTIONS . . . . . . . . . . 4-10

4.3.1 MainWindow (MAINWIND. FRM) . . . . . . . . . . 4-10

4.3.2 PrefWindow (PREFWIND.FRM) . . . . . . . . . 4-15

4.3.3 ItemEdit Window (ITEMEDIT.FRM) . . . . .... 4-16

4.3.4 RelTable Window (RELTABLE. FRM) . . . . . . . . . 4-19

4.3.5 0ldViews Window (OLDVIEWS. FRM) . . . . . . . . . 4-20

4.3.6 SearchWindow (SEARCHWI.FRM) . . . . . . . . . . 4-21

4.3.7 frmSingleQuery (FRMSQRY.FRM) . . . . . . . 4-23

4.3.8 MultiSearch (MULTSRCH.FRM) . . . . . . . . . . . . . . 4-25

4.3.9 AddRelations Window (ADDRELAT.FRM) . . . . . . . . . . 4-27

4.3.10 InheritRelations Window (INHERITR. FRM) . . . . . 4-29

4.3.11 CustomViews Window (CUSTOMVI.FRM) . . . . . . . 4-30

4.3.12 ChangeWindow (CHANGEWI.FRM) . . . . . . . 4-31

4.3.13 AttributeChanges Record details window, ARecord

(ARECORD.FRM) ................ . . . . . . . . .

4.3.14 ElementChanges Record details window, ERecord

(ERECORD.FRM) . . . . . . . . . . . . 4-36

4.3.15 RelationChanges Record details window, RRecord

(RRECORD.FRM) . . . . . . . . . . . 4-37

4.3.16 HistoryWindow (HISTORYW.FRM) . . . . . . . . . 4-39

4.3.17 History Attribute Record window (HARECORD. FRM) . . . 4-40

4.3.18 History Element Record window (HERECORD.FRM) . . . . . 4-41

4.3.19 History Relation Record window (HRRECORD.FRM) . . . . . 4-42

4.3.20 SelectCompare Window (SELECTCO.FRM) . . . . . . . 4-43

4.3.21 Compare Window (COMPARE. FRM) ......... . 4-44

4.4 SUPPORT ROUTINE MODULE DESIGN DESCRIPTIONS . . . . . . . . . . . 4-44

4.4.1 Global Variables and Constants (GLOBALS.BAS) . . . . 4-45

4.4.2 Initialization Routines (INITIAL.BAS) . . . . . . 4-45

4.4.3 Database Structure Initialization Routines

(INITSTRU.BAS) . . . . . . . . . . . 4-46

4.4.4 Record Editing Rout ines (EDITING.BAS) . . . . . 4-47

4.4.5 Search Routines (SEARCH.BAS) . . . . . . 4-50

4.4.6 Data Export Routines (EXPORT.BAS) . . . . . . . 4-51

4.4.7 Edit Menu Routines (EDITMENU.BAS) . . . . . . . 4-52

4.4.8 Miscellaneous Utility Routines (UTILITY.BAS) . . . . . 4-53

4.4.9 Redlining routines (REDLINE. BAS) . . . . . . . 4-56

4.4.10 Additional search query routines (QUERY. BAS) . . . . 4-57 
WHC-SD-WM-SDR-012 Rev. 0

5.0 REFERENCES ...................... . . . . .

APPENDIX A SUBROUTINE AND FUNCTION LIST . . . . . . . . . . . . . A-1

LIST OF TABLES

1. Event Procedure Definitions . . . . . . . . . . . . . . . 4-2

2. Element Tables ....................... . 4-3

3. Element Attributes ................... . . . . 4-4

4. Relation Tables . . . . . . . . . . . . . . . . . . 4-6

5. Relation Table Attributes . . . . . . . . . . . . . . . . . . 4-7

6. ElementChanges Table Attributes . . . . . . . . . . . . . . . . . 4-8

7. AttributeChanges Table Attributes . . . . . . . . . . . . . . . . 4-8

8. RelationChanges Table Attributes . . . . . . . . . . . . . 4-9 


\subsection{INTRODUCTION}

Systems Engineering (SE) information for the Tank Waste Remediation System (TWRS) is currently stored and managed with the RDD- $100^{1}$ software application. The RDD-100 application manages data supporting the systems engineering process including functions, requirements, and architecture definitions for specifications. The RDD-100 application runs on a UNIX workstation, costs over $\$ 30 \mathrm{~K}$ per 1 icense, and is not an open system. Therefore, a personal-computer (PC)-based system was developed to increase the ability of engineers and managers on-site to access and interact with the information stored in the RDD-100 system. To provide a more open and costeffective access to the RDD-100 data for a wide range of users, a relational database with a graphical user interface (GUI) was developed for PC users running Microsoft Windows. This software application is titled, "Requirements Management System Browser" (RMSB) or "Browser".

The Browser data structure and interface was designed to closely mimic the RDD-100 data structure and terminology. The database tables were designed to match each element type in RDD-100 and the relationships between elements were recorded in a table named after the RDD-100 relationship. To transfer data from RDD-100 to the Browser, a PC-based GUI was developed titled - RMSB Data Loader (RMSB-DL) or the "Parser". The Parser reads and parses an RDD-100.rdt file and loads the RMSB database.

${ }^{1} \mathrm{RDD}-100$ is a registered trademark of Ascent Logic Corporation 
WHC-SD-WM-SDR-012 Rev. 0

This page intentionally left blank. 


\subsection{PURPOSE AND SCOPE}

The purpose of this document is to provide an "as-built" design description for the RMSB application commonly referred to as the "Browser". This includes a specification of the database structures and the GUI. The description contains sufficient detail to describe the processes used by the software. This document does not include the Parser, the RMSB installation software, or the RMSB updating software. Installation and update of the RMSB application is covered in the documentation on the TWRS SE Tools generic installation program and generic automatic update software. Revisions of the software are covered by the draft TWRS Systems Engineering Software Configuration Management PIan (WHC-SD-WM-CSCM-034) for the TWRS SE Tools. 
WHC-SD-WM-SDR-012 Rev. 0

This page intentionally left blank. 


\subsection{ABBREVIATIONS AND ACRONYMS}

The table below identifies and defines the abbreviations and acronyms used within this document.

\begin{tabular}{|c|c|}
\hline $\begin{array}{l}\text { Acronym } \\
\text { ERA } \\
\text { GUI } \\
\text { RMACS } \\
\text { RMSB } \\
\text { RMSB-CMU } \\
\text { RMSB-DL } \\
\text { SE } \\
\text { TWRS }\end{array}$ & $\begin{array}{l}\text { Definition } \\
\text { Element-Relation-Attribute, the database model used } \\
\text { Graphical User Interface } \\
\text { Requirements Management and Assured Compliance System } \\
\text { Requirements Management System Browser, the Browser } \\
\text { RMSB Change Management Utility } \\
\text { RMSB Data Loader, the Parser } \\
\text { Systems Engineering } \\
\text { Tank Waste Remediation System }\end{array}$ \\
\hline
\end{tabular}


WHC-SD-WM-SDR-012 Rev. 0

This page intentionally left blank. 


\subsection{DESIGN DESCRIPTION}

This section of the document describes the design of the Browser. The section on database structure is also applicable to the Parser. Section 4.1 includes background information on Visual Basic ${ }^{2}$, which is necessary to understand most aspects of the design description. The database structures used by the software are described in Section 4.2. Section 4.3 describes the design of the various forms (.FRM files) that make up the software application, including objects and events associated with each form, and navigation to other forms. Section 4.4 overviews the various Visual Basic subroutine files (.BAS files) to be developed, and describes some of the details of more complex routines and functions. Sections 4.3 and 4.4 contains "pseudo-code" to identify the procedural or functional steps for the various event procedures and supporting routines.

\subsection{BACKGROUND INFORMATION}

The development of the software was done with Visual Basic Professional edition, version 3.0. Visual Basic applications are built starting with window forms that have controls (buttons, tables, menus) placed on them. Each of the forms and controls have event procedures that can be used for input, output, or processing. This leads to a program structure that is more objectoriented than procedural or functional. Because of the object-oriented paradigm, the suggested format for the Software Design Description, which is found in WHC-CM-3-10, Software Practices, Appendix H, is not followed. This document is organized around the forms/windows (.FRM files) that are used to develop the GUI. Support routines and functions are organized into Basic modules (.BAS files) that can be called from any form.

Visual Basic also does not have parameter passing mechanisms between window forms. For this reason, all of the table pointers and many of the variables and constants in the software are defined globally. All global declarations for variables and constants are defined in one file, GLOBALS.BAS, which is automatically loaded when the application is run. The Ma in Window (MAINWIND. FRM) is also automatically loaded as the first window displayed by the software.

Table 1 identifies the various event procedures that are automatically created when windows and controls are created. Initially, the source code that is defined for each of these event procedures is empty. The software developer enters the appropriate Visual Basic code to be executed for the desired events. The table also identifies which object types (forms, controls, etc.) use the event procedures. There are additional event procedures for each object type, however, Table 1 identifies those events that are most commonly used in the software.

2 Visual Basic is a registered trademark of Microsoft Corporation. 
Table 1. Event Procedure Definitions.

\begin{tabular}{|l|l|l|}
\hline \multicolumn{1}{|c|}{$\begin{array}{c}\text { Event } \\
\text { Procedure }\end{array}$} & \multicolumn{1}{|c|}{ Object Types } & \multicolumn{1}{c|}{ Description } \\
\hline Load & Forms & $\begin{array}{l}\text { This event is triggered the first time a } \\
\text { form is displayed }\end{array}$ \\
\hline Unload & Forms & $\begin{array}{l}\text { This event is triggered when a form is } \\
\text { resized either by the user or within code }\end{array}$ \\
\hline Click & $\begin{array}{l}\text { Forms, Most } \\
\text { Control Types } \\
\text { closed }\end{array}$ & $\begin{array}{l}\text { This event is triggered when a form or } \\
\text { control is clicked by a mouse button } \\
\text { press (usually only left mouse button) }\end{array}$ \\
\hline Dblclick & $\begin{array}{l}\text { Forms, Lists, } \\
\text { Tables, Labeis, } \\
\text { Pictures }\end{array}$ & $\begin{array}{l}\text { This event is triggered when a form or } \\
\text { control is double-clicked with the mouse } \\
\text { (usually only left mouse button) }\end{array}$ \\
\hline Validate & $\begin{array}{l}\text { Data Aware } \\
\text { Controls }\end{array}$ & $\begin{array}{l}\text { This event is triggered during the } \\
\text { validation phase on a database record } \\
\text { associated with a data aware control }\end{array}$ \\
\hline Reposition & $\begin{array}{l}\text { Data Aware } \\
\text { Controls }\end{array}$ & $\begin{array}{l}\text { This event is triggered when a reposition } \\
\text { on the database table associated with a } \\
\text { data aware control occurs }\end{array}$ \\
\hline Change & $\begin{array}{l}\text { Text Boxes, List } \\
\text { Boxes }\end{array}$ & $\begin{array}{l}\text { This event occurs when a change to the } \\
\text { data within a control is made, typically } \\
\text { by the user entering a new value. }\end{array}$ \\
\hline
\end{tabular}

\subsection{DATABASE STRUCTURE DESIGN DESCRIPTION}

This section identifies the design structure for the database tables used within the software. For this discussion, the database structures are organized into three groups: element tables, relation tables, and change tables. All database tables are in Microsoft Access format. This format is used because Visual Basic provides a direct interface to the Access "jet engine" through built-in functions and routines. The database structure is the same for both the Browser and the Parser.

\subsubsection{El ement Tables}

For each element type currently used in RDD-100, one element table is created for the Browser and the Parser. Each element table has fields that correspond to the attributes of the RDD-100 elements. Note that the elements, attributes, and relations used within RDD-100 will likely change during the 1 ife of the software. Table 2 identifies the element tables that are used within the Browser, and the corresponding RDD-100 and RMACS nomenclature. 
Table 2. Element Tables.

\begin{tabular}{|l|l|l|}
\hline \multicolumn{1}{|c|}{ Element Table Name } & \multicolumn{1}{|c|}{ RDD Element Name } & \multicolumn{1}{c|}{ RMACS Element Name } \\
\hline Architecture & Component & Architecture \\
\hline Category & Category & Category \\
\hline Decision & Decision & Decision \\
\hline Function & TimeFunction & Function \\
\hline Input/Output & TimeItem & Input/0utput \\
\hline Issue & Critical Issue & Issue \\
\hline Note & Comment & Note \\
\hline Organization & Organization & Organization \\
\hline Required Analysis & RequiredAnalysis & Required Analysis \\
\hline Requirement & SystemRequirement & Requirement \\
\hline Source & Source & Source \\
\hline Engineer & Engineer & Engineer \\
\hline Interface & Interface & Interface \\
\hline ItemLink & ItemLink & ItemLink \\
\hline
\end{tabular}

Each of the element types listed in Table 2 has a set of attributes associated with it. These attributes correspond to the fields of each element record in the database. A number of the attributes are used by all element types and are known as the "common" or "core" attributes. Table 3 identifies alf of the attributes to be used in the software, both the common and elementspecific attributes. 
Table 3. Element Attributes.

\begin{tabular}{|c|c|c|c|}
\hline Attribute & $\begin{array}{l}\text { Data } \\
\text { Type } \\
\end{array}$ & $\begin{array}{l}\text { Size, } \\
\text { Bytes }\end{array}$ & Description \\
\hline Name & String & 250 & $\begin{array}{l}\text { The unique name of the element record, } \\
\text { which cannot be empty. Common to a11 } \\
\text { element types. }\end{array}$ \\
\hline Number & String & 50 & $\begin{array}{l}\text { Number, sometimes hierarchical. Common } \\
\text { to all element types. }\end{array}$ \\
\hline Creator & String & 40 & $\begin{array}{l}\text { Creator of the record in RDD- } 100 . \\
\text { Common to all element types. }\end{array}$ \\
\hline CreationDate & Date & 8 & $\begin{array}{l}\text { Date the record was created in RDD- } 100 \text {. } \\
\text { Common to all element types. }\end{array}$ \\
\hline ModificationDate & Date & 8 & $\begin{array}{l}\text { Date the record was last modified in } \\
\text { RDD- } 100 \text {. Common to all element types. }\end{array}$ \\
\hline ModificationTime & Time & 8 & $\begin{array}{l}\text { Time the record was last modified in } \\
\mathrm{RDD}-100 \text {. Common to al1 element types. }\end{array}$ \\
\hline Description & Memo & $32 \mathrm{~K}$ & $\begin{array}{l}\text { The description associated with the } \\
\text { element record. Common to all element } \\
\text { types. }\end{array}$ \\
\hline Color & Integer & 4 & $\begin{array}{l}\text { Color of the element, if a change has } \\
\text { occurred. Common to all element types. }\end{array}$ \\
\hline Status & List 6 & 25 & The status of a Decision record. \\
\hline Choice & Memo & $32 k$ & $\begin{array}{l}\text { The choice, or description of how a } \\
\text { choice was made for a Decision. }\end{array}$ \\
\hline Alternatives & Memo & $32 K$ & $\begin{array}{l}\text { The alternatives considered for a } \\
\text { Decision. }\end{array}$ \\
\hline IssueType & List 1 & 20 & The type of Issue record. \\
\hline Actua1Date & Date & 8 & $\begin{array}{l}\text { The actual date of the resolution of an } \\
\text { issue. }\end{array}$ \\
\hline Priority & List 2 & 15 & The priority of an issue. \\
\hline ScheduledDate & Date & 8 & $\begin{array}{l}\text { The scheduled date for the resolution } \\
\text { of an issue. }\end{array}$ \\
\hline Status & List 4 & 10 & The status of a Requirement. \\
\hline Requirement Type & List 5 & 15 & The type of Requirement. \\
\hline ApprovedBy & String & 100 & Who approved the Requirement. \\
\hline SourceType & List 3 & 25 & The document type for a Source. \\
\hline
\end{tabular}


As noted in Table 3 , some attributes are of an enumerated type (i.e., there is a finite list of values that are allowed for the attribute). The lists referenced in Table 3 are shown below. Note that the value "[nil]" means that the value contains the empty string.

List 1: IssueType List

[nil]

Issue

Required Analysis

List 2: Priority List

[nil]

A (Very High)

B (High)

C (Medium)

D (Low)

List 3: SourceType List

[nil]

Change Request

Meeting Minutes

Originating Requirements

other

Project Memo

Standard

Trade-off Study Report

List 4: Requirement Status List

[nil]

Approved

Changed

Pending

Rejected

List 5: RequirementType List

[nil]

Design

Information

List 6: Decision Status List

[nil]

Open

Resolved

Enabling Assumption

The Element tables also have two indexes. The first index, "Name Index" is an index on the "Name" attribute of a table. The "Number Index" is an index on the "Number" and "Name" attributes of a table. These indexes are used for faster searching and sorting of the element tables. 


\subsubsection{Relation Tables}

Within RDD-100, elements records are "linked" to each other using various relations. For example, a Source element documents a Requirement or Decision element. Al1 relations are bi-directional, so that every relation has a converse relation. For example, the converse of the documents relation is the relation documented by. Table 4 identifies all of the relation pairs currently used in RDD-100 that are implemented in the Browser and Parser software.

Table 4. Relation Tables.

\begin{tabular}{|c|c|}
\hline Relation Table and RDD Name & Description \\
\hline analyzed_by / analyzes & $\begin{array}{l}\text { A RequiredAnalysis analyzes a } \\
\text { Decision or Issue }\end{array}$ \\
\hline annotated_by / annotates & $\begin{array}{l}\text { A Note annotates any other element } \\
\text { type }\end{array}$ \\
\hline built_from / built_in & $\begin{array}{l}\text { Defines a hierarchy of Architecture } \\
\text { elements }\end{array}$ \\
\hline categorized_by / categorizes & $\begin{array}{l}\text { A Category categorizes any other } \\
\text { element type }\end{array}$ \\
\hline documented_by / documents & $\begin{array}{l}\text { A Source documents any other element } \\
\text { type }\end{array}$ \\
\hline incorporated_by / incorporates & $\begin{array}{l}\text { Defines a hierarchy of Requirement } \\
\text { elements }\end{array}$ \\
\hline input_to / inputs & $\begin{array}{l}\text { An Input/Output is input_to a } \\
\text { Function }\end{array}$ \\
\hline output_from / outputs & $\begin{array}{l}\text { An Input/Output is output_from a } \\
\text { Function }\end{array}$ \\
\hline performed by / performs & An Architecture performs a Function \\
\hline primary_is / primary_for & $\begin{array}{l}\text { An Organization is primary_for any } \\
\text { element type }\end{array}$ \\
\hline secondary_is / secondary_for & $\begin{array}{l}\text { An Organization is secondary_for any } \\
\text { element type }\end{array}$ \\
\hline traced_from / traces_to & $\begin{array}{l}\text { A Decision, Issue, or Requirement } \\
\text { traces to any element type }\end{array}$ \\
\hline owned by / owns & An Engineer owns any element type \\
\hline contains / is contained by & An Interface contains an ItemLink \\
\hline carries / carried by & An ItemLink carries an Input/0utput \\
\hline
\end{tabular}


For each of the relations listed in Table 4, a relation table is created with the name of the relation. All relation tables have the same fields, as identified below in Table 5. All relation tables have two indexes, the first being the "Source Index" on the source element type and name, and a "Related Index" on the related element type and name.

Table 5. Relation Table Attributes.

\begin{tabular}{|l|l|r|l|}
\hline \multicolumn{1}{|c|}{ Attribute } & Data Type & \multicolumn{1}{|c|}{ Size } & \multicolumn{1}{c|}{ Description } \\
\hline Source Type & Integer & 4 & $\begin{array}{l}\text { The element type of the source } \\
\text { element of the relation. }\end{array}$ \\
\hline Source Element & String & 250 & The name of the source element. \\
\hline Source Number & String & 50 & The number of the source element. \\
\hline Related Type & Integer & 4 & $\begin{array}{l}\text { The element type of the related, or } \\
\text { target, element of the relation. }\end{array}$ \\
\hline Related Element & String & 250 & The name of the related element. \\
\hline Related Number & String & 50 & The number of the related element. \\
\hline Color & Integer & 4 & The color for a changed relation. \\
\hline
\end{tabular}

\subsubsection{Change Tables}

To generate "delta" files for RDD-100, the Browser software retains information on all of the changes made by the user. Changes are organized into three categories: element changes, attribute changes, and relation changes. Element changes include the creation, renaming, or deleting of element records. Attribute changes are changes to the attributes of an existing element. Relation changes occur when relation links are created or deleted between elements.

Table 6 identifies the attributes for the ElementChanges table. The ElementChanges table has the following indexes:

ChangeIndex: ChangeType field

DateTimeIndex: ModificationDate and ModificationTime fields

NewName Index: NewElementName field

PrimaryKey: ElementType and ElementName fields

StatusIndex: Status field 
Table 6. ElementChanges Table Attributes.

\begin{tabular}{|l|l|r|l|}
\hline \multicolumn{1}{|c|}{ Attribute } & Data Type & \multicolumn{1}{c|}{ Size } & \multicolumn{1}{c|}{ Description } \\
\hline ElementType & Integer & 4 & The element type of the change. \\
\hline ElementName & String & 250 & The name of the changed element. \\
\hline ChangeType & String & 6 & $\begin{array}{l}\text { The type of change to the element } \\
\text { (Create, Rename, or Delete) }\end{array}$ \\
\hline NewElementName & String & 250 & The new element name if a Rename. \\
\hline ModificationDate & Date & 8 & The date the change was made. \\
\hline ModificationTime & Date & 8 & The time the change was made. \\
\hline ModifiedBy & String & 50 & Who made the change. \\
\hline Status & String & 20 & The status of the change. \\
\hline Justification & Memo & $32 \mathrm{~K}$ & $\begin{array}{l}\text { The justification or reason for the } \\
\text { change. }\end{array}$ \\
\hline
\end{tabular}

The AttributeChanges table is used to retain information associated with data changes to the attributes of elements. The fields of the AttributeChanges table are shown below in Table 7 . The Attributechanges table has the three indexes given below.

DateTimeIndex: ModificationDate and ModificationTime fields PrimaryKey: ElementType and ElementName fields

StatusIndex: Status field

Table 7. AttributeChanges Table Attributes.

\begin{tabular}{|l|l|r|l|}
\hline \multicolumn{1}{|c|}{ Attribute } & Data Type & \multicolumn{1}{c|}{ Size } & \multicolumn{1}{c|}{ Description } \\
\hline ElementType & Integer & 4 & The element type of the change. \\
\hline ElementName & String & 250 & The name of the changed element. \\
\hline Changes & Integer & 4 & The attribute modified. \\
\hline NewValue & Memo & $32 \mathrm{~K}$ & The new value for the attribute. \\
\hline ModificationDate & Date & 8 & The date the change was made. \\
\hline ModificationTime & Date & 8 & The time the change was made. \\
\hline ModifiedBy & String & 50 & Who made the change. \\
\hline Status & String & 20 & The status of the change. \\
\hline Justification & Memo & $32 \mathrm{~K}$ & $\begin{array}{l}\text { The justification or reason for the } \\
\text { change. }\end{array}$ \\
\hline
\end{tabular}


The RelationChanges table identifies all relations that have been inserted or removed between element records. Table 8 identifies the attributes of the Relationchanges table. Below are the indexes used for this tabie.

ChangeIndex: ChangeType field

DateTimeIndex: ModificationDate and ModificationTime fields

SourceIndex: SourceType and SourceName fields

Status Index: Status field

TargetIndex: TargetType and TargetName fields

Table 8. Relationchanges Table Attributes.

\begin{tabular}{|c|c|c|c|}
\hline Attribute & Data Type & Size & Description \\
\hline RelationTable & Integer & 4 & Index to the relation table changed. \\
\hline ChangeType & String & 6 & $\begin{array}{l}\text { The type of change made to the } \\
\text { relation (Insert or Remove). }\end{array}$ \\
\hline SourceType & Integer & 4 & $\begin{array}{l}\text { The element type of the source } \\
\text { element changed. }\end{array}$ \\
\hline SourceName & String & 250 & $\begin{array}{l}\text { The name of the source element } \\
\text { changed. }\end{array}$ \\
\hline TargetType & Integer & 4 & $\begin{array}{l}\text { The element type of the target } \\
\text { (related) element changed. }\end{array}$ \\
\hline TargetName & String & 250 & $\begin{array}{l}\text { The name of the target (related) } \\
\text { element changed. }\end{array}$ \\
\hline ModificationDate & Date & 8 & The date the change was made. \\
\hline ModificationTime & Date & 8 & The time the change was made. \\
\hline ModifiedBy & String & 50 & Who made the change. \\
\hline Status & String & 20 & The status of the change. \\
\hline Justification & Memo & $32 K$ & $\begin{array}{l}\text { The justification or reason for the } \\
\text { change. }\end{array}$ \\
\hline
\end{tabular}




\subsection{FORM (WINDOW) DESIGN DESCRIPTIONS}

This section discusses the design of each of the windows (.FRM files) associated with the software. Note that this does not include message boxes, which are provided by Visual Basic, file open, and save dialogue boxes, or other simple windows such as the Welcome window. Pseudo-code for event procedures for forms and controls are described in this section as well. Note that many of the controls are named by the function they perform rather than the actual implemented name of the control. For example, a Print button may have an actual name of IEPrint. The actual name of the control is 1isted between square brackets, for example [IEPrint]. Routines or functions called by the various events are also listed between square brackets. These routine names and their arguments can be found in Appendix $A$.

The following forms are not discussed because they are fairly simple and have very little source code associated with them:

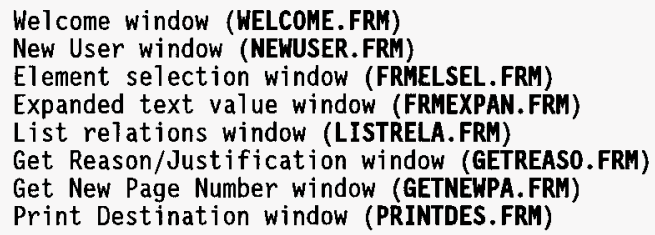

\subsubsection{MainWindow (MAINWIND.FRM)}

The form MainWindow is the main window of the software. This is also the window that is automatically displayed when the program begins execution. This is a resizable window that is dominated by a table of elements of a currently selected type. Most of the command menus are on the MainWindow. Below is a brief description of each of the items for the window, and any nontrivial event procedures associated with the items.

Menus: The Browser MainWindow has the following menus:

File Menu

Open Database: Open any Browser database file Repair Database: Attempt to repair current database file Preferences: Display the Preferences window Export: Menu for exporting (has submenus)

Changes to RDD-100: Export changes in . RDT format

Changes to RMSB: Export changes to RMSB-CMU

Changes to Text: Export changes as ASCII text

F\&R Matrix: Matrix of functions and requirements

Record Count Report: Number of records for each table Erase History: Erase a11 change history

Exit: Exit from the Software 
Edit Menu (Used for all windows with Edit menu)

Cut: Cut information to clipboard

Copy: Copy information to clipboard

Append: Copy and append information to clipboard

Paste: Paste information from clipboard

Select All: Select all text in current control

Display Clipboard: Display the text contents of the clipboard

Records Menu

Add New: Add a new record of the selected type

Delete: Delete a set of selected records

View Changes: View change information (display Changes window)

Query Menu:

Single Table: Displays single table search window frmsinglequery Two Table Search: Displays two table search window MultiSearch Multiple Tables: Displays SearchWindow

Help Menu

Using Help: Help on using the Microsoft WinHelp $p^{3}$ software Contents: Table of Contents

General Info: General information (overview)

Edit Menu: Information on the Edit menu

Main Window: Information on the MainWindow

Menus: Information on the MainWindow menus

Buttons: Information on the Mainwindow buttons

Database Structure: Information on the database structure

This Element: Information on the current element type

About: Version information for the Browser software

Data Version: Version information on the current database file

Change History: Browser software change history information

Menu Event Procedures: The following are the events associated with each of the menus of the Mainwindow as listed above.

File Menu, Open Database [mnuFileopen]

Get file name to open from the user

Open new file name [ChangeDatabase]

File Menu, Repair Database [mnuRepair]

Query the user if they really want to repair the database

Close the current database [RepairTheDatabase]

Use the Visual Basic RepairDatabase command [RepairTheDatabase]

Re-open the current database [RepairTheDatabase]

File Menu, Preferences [mnuPrefs]

Show the preferences window Prefwindow

File Menu, Export - Changes to RDD-100 [mnuSupRDT]

Get output file name from the user

Export . RDT file as a subordinate [ToSuperiorRDT]

${ }^{3}$ WinHelp is a registered trademark of Microsoft Corporation. 
File Menu, Export - Changes to RMSB [mnuExportRMSB]

Export change tables to empty database [ExportRMSB]

File Menu, Export - Changes to Text [mnuFECtoText]

Get output file name from the user

Export changes as ASCII Text [ToASCIIText]

File Menu, Export - F\&R Matrix [mnuFRMatrix]

Get output file name from the user

Export Functions and Requirements Matrix [ExportMatrix]

File Menu, Export - Record Count Report [mnuDBReport]

Get output file name from the user

Print record counts to file [DBReport]

File Menu, Erase History [mnuMainErase]

Query the user if they really want to erase all history

Erase all change records in the database

Set the "color" of all elements to unchanged (black)

Refill the MainWindow table [RefillMain]

File Menu, Exit [mnuExit]

Close the currently open database file [CloseTheDatabase]

Save the user's preferences [SaveUserinfo]

Exit the software

Edit Menu, cut [mnuMaincut]

Cut the selected text to the Windows clipboard [CutToclipboard]

Edit Menu, Copy [mnuMainCopy]

Copy the selected text to the Windows clipboard [CopyToClipboard]

Edit Menu, Append [mnuMwAppend]

Append the selected text to the Windows clipboard [AppendToClipboard]

Edit Menu, Paste [mnuMainPaste]

Paste the selected text from the Windows clipboard [PasteFromClipboard]

Edit Menu, Select A11 [mnuMainSelectA11]

Select all text in the current control [SelectA11Text]

Edit Menu, Display Clipboard [mnuMWDC]

Display current contents of the Windows clipboard [ShowClipboard]

Records Menu, Add New [mnuMainNew]

Set NewRecord flag to true and display empty record [ShowElement]

Records Menu, Delete [mnuMainDelete]

Query user if want to be queried for more than 1 record deleted Delete all records selected (query user where applicable)

Update the MainWindow table 
Records Menu, View Changes [mnuShowChanges]

Display the Changewindow

Query Menu, Sinqle Table [mnuMWQSingle]

Get element type for search from user

Display single table search window frmsinglequery

Query Menu, Two Table Search [mnuTwoTable]

Display two table search window MultiSearch

Query Menu, Multiple Tables [mnuMWQMultiple]

Get element type for search from user

Display multiple table search window Searchwindow

Help Menu, Using Help to This Element, and Change History commands

Use WinHelp with the appropriate topic number [Get Help]

Help Menu, About [mnuAbout]

Display the Welcome window

Help Menu, Data Version [mnuDataVersion]

Display message box with current data version information

Controls: The following are the controls associated with the Mainwindow.

ElementList List Box: Select the element type to display

ElementGrid Table: Display the Name and Number fields in a table

SortButton Button: Select the sorting order for the table

Relations Button [RelationsB]: Display the RelWindow for relations

01dViews Button [Commandl]: Display the 01dViews window

Print Button [IEPrint]: Print current table

Exit Button [QuitButton]: Exit the software

FirstPage Button [Mh3dButn1(0)]: Move to first Virtual Page

PreviousPage Button [Mh3dButn1(1)]: Move to the previous Virtual Page

NextPage Button [Mh3dButn1(2)]: Move to the next Virtual Page

LastPage Button [Mh3dButn](3)]: Move to the last Virtual Page

Pagelabel Text Box: Display current Virtual Page

Form and Control Event Procedures: The following are the events associated with the MainWindow form and the controls listed above.

Sub Form Load - Triggered when the form is initially loaded

Show Welcome window

Initialize program parameters [InitProgram]

Sub Form Resize - Triggered when the form is resized by user or software Check for minimum window width

Resize ElementGrid table

Align control buttons at bottom of window

Sub Form LinkExecute - Triggered on a Dynamic Data Exchange (DDE) "Execute" command

Parse the DDE Link command and handle accordingly (show, quit, or error) 
Sub ElementList Click - User selects new item in the ElementList Set new Current Element Type

Refill ElementGrid with records from new element [UpdateMainGrid]

Sub ElementGrid DblClick - User double-clicks on a table row/record Determine record clicked

If record deleted, then display warning and ignore event

Otherwise, display the selected record [ShowElement]

Sub SortButton Click - User clicks on the "Sort by Name/Number" button

Sort and redisplay the ElementGrid by Name or Number [UpdateMainGrid]

Sub RelationsB Click - User clicks on the "Relations" button

Display the relations window RelWindow

Sub 01dViews C1ick [Command1] - User clicks on the "01d Views" button Display the oldViews window

Sub IEPrint Click - User clicks on the "Print" button

Determine format to print (labeled text or two columns)

Print Number and Name fields for all rows

Sub QuitButton Click - User clicks on the "Exit" button

Close current database file [CloseTheDatabase]

Save user preferences [SaveUserInfo]

Exit software

Sub PageLabel Dbiclick - User double-clicks on the page 1 abel text box

Get virtūal page number from user

Set new top virtual page

Refill main window table [UpdateMainGrid]

Sub FirstPage Click [Mh3dButnl(0)] - User clicks on the "First Page" button Set top virtual page to 1

Refill main window table [UpdateMainGrid]

Sub PreviousPage Click [Mh3dButn1(1)] - User clicks "Previous Page" button Decrement tōp virtual page by 1

Refill main window table [UpdateMainGrid]

Sub NextPage Click [Mh3dButnl(2)] - User clicks "Next Page" button Increment top virtual page by 1

Refill main window table [UpdateMainGrid]

Sub LastPage_Click [Mh3dButnl(3)] - User clicks "Last Page" button Set top virtual page to last page

Refill main window table [UpdateMainGrid] 


\subsubsection{PrefWindow (PREFWIND. FRM)}

The PrefWindow is used to set user preferences for the various software options. The preferences window is displayed when a user clicks on the "Preferences" command of the "File" menu from the MainWindow.

Menus: The PrefWindow form only the "Help" menu.

Menu Event Procedures: Each of the commands of the "Help" menu will display the appropriate on-line help topic.

Controls: The controls of the preferences allow the user to set various program options. The controls used by this window are:

DataView List [List1]: Select viewing mechanism

Sortorder List [List2]: Set sorting order for current element type

NamesView List [List3]: Select nomenclature used for browser

ElementCreate Check Box [Checkl]:Query reason on element create

ElementDelete Check Box [Check2]:Query reason on element delete

ElementModify Check Box [Check3]:Query reason on element modified

Relationcreate Check Box [Check4]:Query reason on relation create

RelationDelete Check Box [Check5]:Query reason on relation delete LastSelected 0ption [0ptionl]:Always show last selected relation

OnlywhenAsked Option [0ption2]: Show only when asked (reset to none)

VMode Check Box: Turns on and off the virtual mode feature

VSize Text Box: Sets the virtual page size for virtual mode

Cance 1 Button [Commandl]: Exit the window without saving changes

OK Button [Command2]: Exit the window and save changes

Form and Control Event Procedures: The following are events associated with the preferences window form and the controls listed above.

Sub Form Load - Triggered when window is first displayed

FilT in the Dataview, Sortorder, and Namesview lists

Set check box values and virtual mode values

Sub Commandl Click - User clicks the "Cancel" button

Unload the window

Sub Command2 Click - User clicks the "OK" button

Update the element detail viewing parameters if changed

Update the nomenclature if changed

Update the sorting order of the main window if changed [UpdateMainGrid]

Set reason/justification query values

Update the virtual mode settings if changed

Unload the window

Sub List1 click - User clicks on the data view list

If "Custom..." was selected, display the Customviews window

Sub VMode $\mathrm{Click}$ - User clicks on the virtual mode check box

EnabTe (Disable) vsize text box 


\subsubsection{ItemEdit Window (ITEMEDIT. FRM)}

The ItemEdit window, often referred to as the "Record Details" window, displays the attributes and relations of a single element record. This window al so allows the user to modify the attributes, create and delete relations, delete the element record, or add a new element record. The window is resizable, and which attributes are displayed is defined from the preferences window, Prefwind, and the Customviews window. The window is also built "onthe-fly" by cloning and placing the appropriate controls so that the source code for the window does not have to be modified when the database structure changes. The window is displayed whenever an element record instance is double-clicked in any of the tables in the other windows, such as the MainWindow or one of the search windows.

Menus: The ItemEdit window has the following menus:

Edit Menu: Same as MainWindow Edit menu

Records Menu

Add New: Add a new element record

Delete: Delete current element record

Add $\operatorname{Relation(s):~Add~relations~to~the~element~}$

Delete Relation(s): Delete selected relations from element

Inherit Relations: Inherit relations from another element

Views Menu

0ld Views: Display the oldViews window

Go to Previous View: Go to the previously displayed record

Change History: Display the change history window Historywindow

Query Menu: Same as MainWindow Query menu

Reports Menu (Wi11 be expanded in the future)

Architecture: Produce an architecture report

Help Menu: Similar to Mainwindow help menu

Menu Event Procedures: The following are the event procedures associated with each of the menus for the ItemEdit window as listed above.

Edit Menu: A1l commands same as MainWindow Edit menu

Records Menu, Add New [mnuNewRecord]

Verify and save the current record information

Create a new blank record for data entry

Records Menu, Delete [mnuRecordDelete]

Query user to delete record

Delete the record [DeleteElement]

$\mathrm{Fill}$ in relations grid with next element [FillElement]

Records Menu, Add Relation(s) [mnuAddRelation]

Verify and save the current record information [CanSave, UpdateElement]

Display the AddRelations window 
Records Menu, Delete Relation(s) [mnuDeleteRelation]

Check validity of rows to delete

Query user if they want to be queried

For each relation:

Determine relation link to delete

Delete the link [RemoveRelation]

Update RelationsGrid

Records Menu, Inherit Relations [mnuIEInherit]

Verify and save the current record information [CanSave, UpdateElement]

Display the InheritRelations window

Views Menu, oldViews [mnuIE01dViews]

Verify and save the current record information [CanSave, UpdateElement]

Display the 0ldViews window

Views Menu, Go to Previous View [mnuIEGoto]

Verify and save the current record information [CanSave, UpdateElement]

Pop the previous view off the view stack

Display the new record [ShowElement, Fillelement]

Views Menu, Change History [mnuIEVCH]

Verify and save the current record information [CanSave, UpdateElement]

Display the change history window [ShowChangeHistory]

Query Menu: Same as MainWindow Query window

Reports Menu, Architecture [mnuArchRept]

Clear the previous architecture report

Determine all related elements to the current architecture

Display the report results (uses Crystal Reports)

Help Menu: Display the appropriate On-Line help topic [Get_Help]

Controls: The following are the controls associated with the ItemEdit window. Note that some of the controls are indexed, so that they can be cloned as needed to build the window on-the-fly.

Attributelabel (Index) Label: Attribute label for any/all attributes Attributelist (Index) List Box: Attribute list for enumerated attributes AttributeMemo(Index) Text Box: Scrollable, multi-line attribute value AttributeValue(Index) Text Box: Single line attribute value box Related Element List [REList]: Element type to show in Relations Grid RelationsGrid Table: Displays the selected related element records Previous View Button [IECance1]: Moves to the previously viewed record First Record Button [IEFirst]: Move to the first record in the table Previous Record Button [IEPrevious]: Move to the previous record Next Record Button [IENext]: Move to the next record in the table Last Record Button [IELast]: Move to the last record in the table old Views Button [IE0ldViews]: Display the 01dViews window Print Button [IEPrint]: Print current record information Return Button [IEReturn]: Exits the window 
Form and Control Event Procedures: The following are the events associated with the ItemEdit form and the controls listed above.

Sub Form Load - Triggered when window is loaded Turn on/off "Records" menu based on Read0nly status

Sub AttributeLabel DblClick(Index) - When an attribute label is double-clicked Display the $0 \bar{n}-$ Line help topic for the selected attribute [Get_Help]

Sub REList Click - When the related element list changes RefilT the RelationsGrid with the related elements [FillifRelGrid]

Sub RelationsGrid DblClick - When user double-clicks on related element Determine vaTidity of element selected Verify and save the current record information [CanSave, UpdateElement] Display the new element record [ShowElement or FillElement]

Sub IEFirst Click - When user clicks on the first record button Verify and save the current record information [CanSave, UpdateElement] Move to the first record in the table Refill the ItemEdit window [FillElement]

Sub IEPrevious $\mathrm{Click}$ - When user clicks on the previous record button Verify and save the current record information [CanSave, UpdateElement] Move to the previous record in the table Refill the ItemEdit window [FillElement]

Sub IENext $\mathrm{Click}$ - When user clicks on the next record button Verify and save the current record information [CanSave, UpdateElement] Move to the next record in the table Refill the ItemEdit window [FillElement]

Sub IELast $\mathrm{Click}$ - When user clicks on the last record button Verif $\bar{y}$ and save the current record information [CanSave, UpdateElement] Move to the last record in the table Refil1 the ItemEdit window [FillElement]

Sub IECancel Click - Same as Views Menu, Go to Previous View

Sub IE01dViews_Click - Same as Views Menu, 01d Views

Sub IEPrint Click - User clicks on the printer icon (print) button Get print destination from user (show PrintDest window) Print information to printer or to a file [PrintRecord, FileRecord] (Note, does not save information first, so they can print without actually making a change to the data)

Sub IEReturn Click - User clicks on the "Return" button Verify and save the current record information [CanSave, UpdateElement] Unload and exit the window 


\subsubsection{RelTable Window (RELTABLE. FRM)}

The relations table window, RelTable, is similar in design and format to the MainWindow form except that the table used by the form is oriented around the relation tables rather than the element tables. In this window, a relation table is selected, and the form's table is filled with all of the element pairs for the selected relation. The window is a resizable window available from the MainWindow.

Menus: The RelTable window has the following menus:

Edit Menu: Same as the Edit menu of the MainWindow

Help Menu: Similar to the MainWindow Help menu

\section{Menu Event Procedures:}

Edit Menu: Same as Edit menu of the Mainwindow

Help Menu: Display the appropriate on-line help information [Get Help]

Controls: The following are controls used by the RelTable form:

RelationList List Box: Select the relation table to display

RelationGrid Table: Display the element pairs of the relation

SortButton Button [SortB]: Select the sorting order for the table

01dViews Button: Display the 01dViews window

Print Button [IEPrint]: Print current table

Return Button [QuitB]: Exit the window

FirstPage Button [Mh3dButnl(0)]: Move to first Virtual Page

PreviousPage Button [Mh3dButnl(1)]: Move to the previous Virtual Page

NextPage Button [Mh3dButn1(2)]: Move to the next Virtual Page

LastPage Button [Mh3dButn1(3)]: Move to the last Virtual Page

Pagelabel Text Box: Display current Virtual Page

Form and Control Event Procedures: The following are the events associated with the MainWindow form and the controls listed above.

Sub Form Load - Triggered when the window is loaded

Initialize the RelationGrid [InitRelGrid]

Sub Form Resize - Triggered when window is resized by user or by software Check for minimum window width

Resize RelationGrid table

Align control buttons at bottom of window

Sub RelationList Click - User clicks on relation list box

Set new current relation table

Refill Relationgrid with records from new element [UpdateRelGrid]

Sub RelationGrid DblClick - User double-clicks on relation grid record

Determine eTement record clicked

If record deleted, then display warning and ignore event

Otherwise, display the selected record [ShowElement] 
Sub SortB Click - User clicks on the "Sort" button

Sort ${ }^{-}$and redisplay the RelationGrid by source or target [UpdateRelGrid]

Sub 01dViews Click - User clicks on the "0ld Views" button

Display the 0ldViews window

Sub IEPrint Click - User clicks on the "Print" button

Print Element Type, Number and Name fields for all rows

Sub QuitB Click - User clicks on the "Return" button

UnTōad and exit the RelTable window

Sub PageLabel Dblclick - User double-clicks on the virtual page 1 abel box Get virtūal page number from user

Set new top virtual page

Refill RelationGrid [UpdateRe]Grid]

Sub FirstPage Click [Mh3dButnl(0)] - User clicks "First Page" button Set top virtual page to 1

Refill RelationGrid [UpdateRelGrid]

Sub PreviousPage_Click [Mh3dButn1(1)] - User clicks "Previous Page" button Decrement tōp virtual page by 1

Refill Relationgrid [UpdateRelGrid]

Sub NextPage C1ick [Mh3dButnl(2)] - User clicks "Next Page" button Increment top virtua? page by 1

Refill RelationGrid [UpdateRelGrid]

Sub LastPage Click() [Mh3dButn](3)] - User clicks "Last Page" button Set top virtual page to last page

Refill RelationGrid [UpdateRelGrid]

\subsubsection{OldViews Window (OLDVIEWS.FRM)}

The 01 dViews window, or Previous Views window, is used to display all of the element records the user has viewed since launching the program. This keeps a running history of records visited. Double-clicking on a previously viewed record will return to that element record. The window is a resizable window that can be accessed from the MainWindow, ItemEdit window, or RelTable window.

Menus: The 0ldViews Window contains the standard "Help" and "Edit" menus.

Menu Event Procedures: The "Help" and "Edit" menu event procedures are similar to the MainWindow menu event procedures.

Controls: The following controls are used with the 0ldviews window:

ViewsGrid Table: Displays previous views

Print Button [IEPrint]: Print views 1ist

clear Button [Command2]: Clears the 1ist

Return Button [Commandi]: Exits the window 
Form and Control Event Procedures: The following are the events associated with the 0ldViews window form and controls listed above.

Sub Form Load - Triggered when form is loaded Initialize the ViewsGrid table

Fill ViewsGrid with a11 views from previous view stack

Sub Form Resize - Triggered when form is resized by user or software Chec̄k for minimum window size

Resize ViewsGrid table and position buttons

Sub ViewsGrid DblClick - User double-clicks on an old view element record Determine element record selected

Display selected element record [ShowElement]

Sub IEPrint_Click - User clicks on the "Print" button Print $\bar{a} 11$ rows to the printer

Sub Command2 Click - User clicks on the "Clear" button Resize and reset view stack size to zero Refresh the ViewsGrid table

Sub Commandl Click - User clicks on the "Return" button Close the oldViews window

\subsubsection{SearchWindow (SEARCHWI.FRM)}

The SearchWindow form was the original window designed to allow the user to do searches on various attributes and relations, in a very flexible way. However, many users have had trouble understanding how to do queries through this window. Subsequently, the frmsinglequery and Multisearch windows were developed (they are discussed below). The SearchWindow is displayed when the Multiple Tables command is selected from the Query menu from either the Mainkindow or ItemEdit window. The window is resizable.

Menus: The Searchwindow has the following menus:

Edit Menu: Same as MainWindow Edit menu Elements Menu Change Search Element Type: Select element type for search Help Menu: Similar to MainWindow Help menu

Menu Event Procedures: The following are the event procedures for the menus shown above.

Edit Menu: Same as MainWindow Edit menu

Elements Menu, Change Search Element Type [mnuSWCST_Click]

Get new element type from user through frmElemsēlect window

Reload the SearchWindow

Help Menu: Display the appropriate on-line help topic [Get_Help] 
Controls: The following controls are found on the SearchWindow:

HistoryGrid Table: Displays the search history as it is built

Attributelist List Box [AttrList]: Attribute or Relation for search

RelTypelist List Box: Relation type selection box

Re]Attrlist List Box: Attribute 1 ist of related element type

ResultsGrid Table: Displays the results of the search

AND Button [Commandl]: Do a search with the conjunction AND

OR Button [Command2]: Do a search with the conjunction OR

Search Button [Command3]: Do the actual search

Reset Button [Command5]: Clear search results

SQL Button [Command6]: Show Structured Query Language (SQL) string

Print Button [IEPrint]: Print the search results

Return Button [Command4]: Exit from the window

Form and Control Event Procedures: The following are the events associated with the SearchWindow form and the controls listed above.

Sub Form Load - Triggered when the window is loaded

Initialize search query strings

Initialize table headers

Initialize AttrList and OpList 1ists

Sub Form Resize - Triggered when the form is resized by the user or software Check for and set minimum window size

Set location of command buttons, resize Historygrid and ResultsGrid

Sub AttrList Click - User clicks on an attribute list item

Determine attribute or relation selected

If attribute selected:

Set operation 1 ist depending on attribute data type

Display Valuelist or ValueText box

Disable related type and related attribute lists

If relation selected:

Enable related type and related attribute lists

Set up operation list depending on related attribute data type

Display Valuelist or ValueText box

Sub RelTypelist Click - User clicks on related element type list item Determine the related element type selected

Refill the RelAttrList list appropriately

Sub RelAttrList Click - User clicks on related element attribute list item Determine the related attribute selected

Fill the OpList with appropriate operations for attribute data type Enable the appropriate ValueList and ValueText boxes

Sub ResultsGrid Dblclick - User double-clicks on record in results grid Determine the record selected

Display the selected record [ShowElement] 
Sub Command1_Click - User clicks on the "AND" button If just completed previous search, add "AND" to search string Otherwise add "AND" to the next search clause [AddToSearch]

Sub Command2 Click - User clicks on the "OR" button If just completed previous search, add "OR" to search string otherwise add "OR" to the next search clause [AddToSearch]

Sub Command3 Click - User clicks on the "Search" button Do the äctual search [AddToSearch]

Sub Command5 Click - User clicks on the "Reset" button Reset search strings

Clear ResultsGrid

Sub Command6 Click - User clicks on the "SQL" button Display the current search SQL string in a MsgBox

Sub IEPrint $\mathrm{Click}$ - User clicks on the "Print" button Print search header and criteria Determine form to use (table or Tabeled text)

Print a11 of the result record information

Sub Command4 Click - User clicks on the "Return" button Unload the Searchwindow

\subsection{7 frmSinglequery (FRMSQRY.FRM)}

This window was developed to provide a simple interface for searching for records within a single element table. The frmsinglequery window is displayed when the Single Tables command is selected from the Query menu from either the MainWindow or Itemedit window. This window was originally developed by Nancy Graves.

Menus: The following menus are available in the frmSinglequery window:

File Menu

Show SQL: Show the current SQL string

Print: Print the search results

Exit: Exit from the window

Edit Menu: Same as the MainWindow Edit menu

Elements Menu: Same as the SearchWindow Elements menu

Sort Menu

By Name: Set sorting order of results to by name

By Number: Set sorting order of results to by number

Help Menu: Similar to the Mainwindow help menu

Menu Event Procedures: The following are the event procedures associated with the menus above.

File Menu, Show SQL [mnuShowSQL]

Build SQL search string and display to the user 
File Menu, Print [mnuPrint]

Create temporary database for search results

Use Crystal Reports to print the search results

File Menu, Exit [mnuExit]

Unload the form and exit the window

Edit Menu: Same as MainWindow Edit menu

Elements Menu: Same as SearchWindow Elements menu

Sort Menu, By Name [mnuSortName]

Set sorting order flag to by name

Sort Menu, By Number [mnuSortNumber]

Set sorting order flag to by number

Help Menu: Similar to MainWindow Help menu

Controls: The following controls are found on the frmsinglequery window

cbooperator(Index) List Box: Lists search operations for each attribute Do Query Button [cmdExecute]: Executes the query

Reset Query Button [cmdReset]: Resets the search results

Display Field Button [cmdEditRecord]: Display long text value

Return Button [cmdClose]: Close and return from the window

Results Grid Table [Gridl]: Displays the search results

Form and Control Event Procedures: The following are the events associated with the frmsinglequery form and the controls listed above.

Sub Form Load - Triggered when window is Toaded

Initiate window parameters and sizes

Initiate attribute controls [LoadControls]

Sub cbooperator Click(Index) - User clicks on an operation list item

Set up the ${ }^{-}$txt Input or listindex boxes depending on operation selected

Sub cmdClose Click - User clicks on the "Return" button

Unload the form and exit from window

Sub cmdEditRecord Click - User clicks on "Display Field" button

Determine record and attribute clicked

Display text value using frmExpand window

Sub cmdExecute Click - User clicks on the "Do Search" button

Create the SQL statement [MakeSQL]

Create snapshot of search results

Fill results grid with results of the search

Sub cmdReset Click - User clicks on the "Reset Query" button

Clear the search results and search results grid 
Sub Gridl Dblclick - User double-clicks on an element record

Determine element record selected

Display the selected element record [ShowElement]

Additional Routines: The following are additional routines defined within the frmsinglequery form.

Sub LoadControls - Loads the various controls for the window

For each attribute of the current element type:

Create a label, operator 1ist, and text or list input box

Fill in the operator list with appropriate operations

Function MakeSQL - Returns the current SQL statement

For each operation that is not "All

Add search clause to the current search strings

\subsubsection{MultiSearch (MULTSRCH.FRM)}

The MultiSearch window was developed to be an easier interface for doing a two table query. The window allows the user to select search criteria on the attributes of two element tables, which are related through a valid relation. The search routine then displays the element records in both tables that met the search criteria and the elements in the two tables are related through a selected relationship. The MultiSearch window is displayed when the Two Table Search command is selected from the query menu from either the MainWindow or ItemEdit window. This window was originally developed by Nancy Graves.

Menus: The following menus are found on the MultiSearch window:

File Menu

Show SQL: Show the current SQL string

Print: Print the search results

Exit: Exit from the window

Edit Menu: Same as the MainWindow Edit menu

Sort Menu

Primary Table Submenu

By Name: Sort primary table results by name

By Number: Sort primary results table by number

Related Table Submenu

By Name: Sort related table results by name

By Number: Sort related table results by number

Help Menu: Similar to the MainWindow help menu

Menu Event Procedures: The following are the event procedures associated with the menu commands above.

File Menu, Show SQL [mnuShowSQL]

Build SQL search string and display to the user

File Menu, Print [mnuPrint]

Create temporary database for search results

Use Crystal Reports to print the search results 
File Menu, Exit [mnuExit]

Unload the form and exit the window

Edit Menu: Same as MainWindow Edit menu

Sort Menu, Primary Table Submenu, By Name [mnuSortName]

Set sorting order flag to by name

Sort Menu, Primary Tab7e Submenu, By Number [mnuSortNumber]

Set sorting order flag to by number

Sort Menu, Related Table Submenu, By Name [mnuSortRByName]

Set sorting order flag to by name

Sort Menu, Related Table Submenu, By Number [mnuSortRByNumber]

Set sorting order flag to by number

Help Menu: Similar to MainWindow Help menu

Controls: The following are controls found on the MultiSearch window:

ElementType(Index) List Box: Element type for primary or related table

Attrlist(Index) List box: Attribute list for primary or related table

Valuelist(Index) List Box: Possible attribute value for either table

ValueText (Index) Text box: Possible attribute search value for table

AND Button [cmdAND]: AND button for search

OR Button [cmdOR]: OR button for search

Reset Button [cmdReset]: Reset the search results

Return Button [cmdReturn]: Unload and exit the window

Search Button [cmdSearch]: Execute the search

ResultsGrid Table: Displays the search results

Form and Control Event Procedures: The following are the event procedures for the Multisrch form and the controls listed above.

Sub Form Load - Triggered when window is loaded

Initialize the window controls and ResultsGrid

Sub ElementList $\mathrm{Click(Index)} \mathrm{-} \mathrm{ElementList} \mathrm{item} \mathrm{is} \mathrm{selected}$

Set the element type based on the Index value

Sub Attrlist click(Index) - Attribute List item is selected

Determin̄e new attribute type selected

Reset and fill the OpList(Index), Valuelist(Index), and ValueText(Index)

Sub cmdAND Click - User clicks the "AND" button

Set the last search operation to "AND"

Sub cmdOR Click - User clicks the "OR" button

Set the last search operation to "OR"

Sub cmdSearch Click - User clicks the "Search" button

Create the SQL query string [MakeSQL] and do the search

Fill the ResultsGrid with results [FillGrid] 
Sub cmdReset c1ick - User clicks the "Reset" button

Clear all of the search values and ResultsGrid

Sub comdReturn Click - User clicks the "Return" button Unload the Multisearch window

Sub ResultsGrid Dblclick - User double-clicks on an element record result Determine the element record selected

Display the element record selected [ShowElement]

Additional Routines: The following are additional routines defined within the MultiSearch form.

Sub ClearGrid - Clears the results grid

Clears and resets the ResultsGrid

Sub Fillarid - Fills the results grid with the results of the search

clear the results grid [ClearGrid]

Build the SQL query string and do the search

For each record in the search results, fill in the ResultsGrid

Sub MakeSQL - Builds the SQL search string

Determine search string for primary search table attributes

Determine relation used between element types

Determine search string for related search table

Build the clauses

\subsubsection{AddRelations Window (ADDRELAT.FRM)}

The AddRelations window is used to add relations to an element record. The window is displayed when the Add Relation(s) command is selected from the Records menu of the ItemEdit window. The user selects the related element type desired, the appropriate relation, and then the records to be related. The window is resizable.

Menus: The AddRelations window contains only the He1p menu.

Menu Event Procedures: The Help menu behaves similar to the Help menu of the MainWindow.

Controls: The following are the controls on the AddRelations window

FirstPage Button [Mh3dButnl(0)]: Move to first Virtual Page PreviousPage Button [Mh3dButn](1)]: Move to the previous Virtual Page NextPage Button [Mh3dButn1(2)]: Move to the next Virtual Page LastPage Button [Mh3dButn](3)]: Move to the last Virtual Page Pagelabel Text Box: Display current Virtual Page Valid Types List Box [VTList]: List of valid related element types Relation List List Box [RList]: List valid relations for element types Related Elements List Box [EL2]: Displays records from related table OK Button [Command2]: Add the selected relation links

Cancel Button [Commandl]: Unload and exit from the window 
Form and Control Event Procedures: The following are the event procedures for the form and the controls listed above.

Sub Form Load - Triggered when the window is loaded

Size and place the controls based on the window size

Add the valid related element types to the VTList list box

Sub Form Resize - Triggered when the window is resized by the user or software Resize element list table EL2 to fit window and place buttons

Sub VTList Click - User clicks on Valid Types list item Determine the relation(s) between selected element types Update the RList with valid relation(s) between elements

Sub RList Click - User clicks on Relation List item (or by software)

Fi11 EL2 table with the records from the selected table

Reset the virtual mode buttons [SetAVButtons]

Sub PageLabel Dblclick - User double clicks on the virtual page number box Get virtūal page number from user

Set new top virtual page

Refill window table [RList_Click]

Sub FirstPage Click [Mh3dButnl(0)] - User clicks the "First Page" button Set top virtual page to 1

Refill window table [RList_Click]

Sub PreviousPage_click [Mh3dButn1(1)] - User clicks "Previous Page" button Decrement tōp virtual page by 1

Refill window table [RList_Click]

Sub NextPage Click [Mh3dButn1(2)] - User clicks the "Next Page" button Increment top virtual page by 1

Refil1 window table [RList_Click]

Sub LastPage_Click [Mh3dButn1(3)] - User clicks the "Last Page" button Set top virtual page to last page

Refil1 window table [RList_Click]

Sub Command2 C1ick - User clicks the "OK" button

Add the selected relations to the current element [AddTheRelations]

Return to the ItemEdit window and refresh grid [FilliERelGrid]

Sub Command1 Click - User clicks the "Cancel" button

Unload and exit the window

Additional Routines: The routines below are defined within the AddRelations window.

Sub SetAVButtons - Sets the status of the virtual mode buttons

Set the visibility and enabled status of the virtual mode buttons 


\subsubsection{InheritRelations Window (INHERITR. FRM)}

The InheritRelations window is used to inherit relations to an element record based on another element record. The window is displayed when the Inherit Relation(s) command is selected from the Records menu of the ItemEdit window. The user selects the related element type desired, the appropriate relation, and then the records to be related. The window is nearly identical to the AddRelations window. The window is resizable.

Menus: The InheritRelations window contains only the Help menu.

Menu Event Procedures: The Help menu behaves similar to the Help menu of the MainWindow.

Controls: The following are the controls on the InheritRelations window

FirstPage Button [Mh3dButnl(0)]: Move to first Virtual Page PreviousPage Button [Mh3dButn1(1)]: Move to the previous Virtual Page NextPage Button [Mh3dButn1(2)]: Move to the next Virtual Page LastPage Button [Mh3dButn1(3)]: Move to the 1ast Virtual Page Pagelabel Text Box: Display current Virtual Page Valid Types List Box [VTList]: List of valid related element types Relation List List Box [RList]: List valid relations for element types Related Elements List Box [EL2]: Displays records from related table OK Button [Command2]: Add the selected relation links

Cancel Button [Command1]: Unload and exit from the window

Form and Control Event Procedures: The following are the event procedures for the form and the controls listed above.

Sub Form Load - Triggered when the window is loaded Size and place the controls based on the window size

Add the valid related element types to the VTList list box

Sub Form Resize - Triggered when the window is resized by the user or software Resize element list table EL2 to fit window and place buttons

Sub VTList_Click - User clicks on a Valid Type list item Determine the relation(s) between selected element types Update the RList with valid relation(s) between elements

Sub RList Click - User clicks on a relation list item (or software) Fi11 EL2 table with the records from the selected table Reset the virtual mode buttons [SetAvButtons]

Sub PageLabe1_DblClick - User double clicks on the virtual page number box Get virtūal page number from user

Set new top virtual page

Refill window table [RList_Click]

Sub FirstPage_Click [Mh3dButnl(0)] - User clicks the "First Page" button Set top virtual page to 1

Refill window table [RList_Click] 
Sub PreviousPage_Click [Mh3dButnl(1)] - User clicks "Previous Page" button Decrement top virtual page by 1

Refill window table [RList Click]

Sub NextPage_click [Mh3dButnl(2)] - User clicks the "Next Page" button Increment top virtual page by 1

Refil] window table [RList_Click]

Sub LastPage_Click [Mh3dButnl(3)] - User clicks the "Last Page" button Set top virtual page to last page

Refil1 window table [RList_click]

Sub Command2 $\mathrm{Click}$ - User clicks the "OK" button Inherit selected relations to the current element [InheritTheRelations]

Return to the ItemEdit window and refresh grid [Fi11IERe1Grid]

Sub Command1 Click - User clicks the "Cancel" button

Unload and exit the window

Additional Routines: The routines below are defined within the InheritRelations window.

Sub SetAVButtons - Set the virtual mode buttons

Set the visibility and enabled status of the virtual mode buttons

\subsubsection{CustomViews Window (CUSTOMVI. FRM)}

The CustomViews window is used to customize the viewing of attributes and relations within the ItemEdit window. The window is displayed when the user clicks on the "Custom..." option for the "Data View" list box on the preferences window, Prefwind. The user can then click on the desired attributes to be displayed or not displayed. The user can save the parameters in a external custom view file (.CVF file). The window is scaled to fit the size of the user's screen.

Menus: The following menus are available in the Customviews window:

File Menu

Open: Open a custom view file

Save: Save settings in current custom view file Save As...: Save settings in a new custom view file

Help Menu: Similar to the MainWindow Help menu

Menu Event Procedures: The following are the event procedures associated with the menu items listed above.

File Menu, Open [mnuCvopen]

Get .CVF file to open with a "File Open" dialogue box

Load the custom view parameters [LoadCustomView]

File Menu, Save [mnuCvSave]

Query user to overwrite .CVF file

Save current view parameters [SaveCurrentView] 
File Menu, Save As...: [mnucvSaveAs]

Get new .CVF file to save with a "File Save" dialogue box

Save the custom view parameters [SaveCurrentView]

Help Menu: Displays the appropriate On-Line help topic [Get_Help]

Controls: The following are the controls used on the Customviews window:

AttributeBox(Index) Check Boxes: Check box to display each attribute DisplayGridBox Check Box: Option to display relations grid

CancelButton Button: Cancels the view changes and exits the window

OKbutton Button: Save the current view changes and exits the window

Form and Control Event Procedures: The event procedures below are used for the form and the controls listed above.

Sub Form Load - Triggered when the window is loaded

Set the width of the window to the size of the screen

Set up the AttributeBox(Index) boxes [DisplaycVAs]

Sub CancelButton Click - User clicks the "Cancel" button

Unload all of the AttributeBox(Index) check boxes

Unload the form

Sub OKButton Click - User clicks the "OK" button

Save the current custom view parameters

Unload all of the AttributeBox(Index) check boxes

Unload the form

Additional Routines: The following are the additional routines defined in the CustomViews window.

Sub DisplaycVAs - Displays the custom views attributes check boxes

For a11 attributes (except Name)

Clone an AttributeBox control and display

Display the DisplayGridBox

\subsubsection{ChangeWindow (CHANGEWI. FRM)}

The Changelindow is used to display all of the changes to the current data file. Note that this is different from the Historywindow that displays all of the changes since the baseline data. As discussed in the database section, changes are organized into three groups: Elements, Attributes, and Relations. The Changewindow will display a summary of the records from one of the three tables, and will display the appropriate detailed information window when a change record is double-clicked. The window is displayed when the View Changes command is selected from the Records menu of the MainWindow. The window is resizable. 
Menus: The Changewindow has the following menus:

Edit Menu: The same as the MainWindow Edit menu

Sort Menu

by Element: Sort the table by element type and name

by Date/Time: Sort table by last modified date (descending)

by Change: Sort table by type of change

by Target: Sort table by target element type (RelationChanges)

Help Menu: Display the appropriate on-line help items

Menu Event Procedures: The following are the event procedures associated with the menus above.

Edit Menu: Same as the MainWindow Edit menu

Sort Menu, by Element [mnuCWSortElement]

Reset the change table sorting index

Refill the change table [RefillACTable, RefilleCTable, RefillRCTable]

Sort Menu, by Date/Time: [mnuCWSortTime]

Reset the change table sorting index to "DateTimeIndex"

Refill the change table [RefillACTable, RefilleCTable, RefillRCTable]

Sort Menu, by Change: [mnuCWSortChange]

Reset the change table sorting index to "ChangeIndex"

Refill the change table [RefilleCTable or RefillRCTable]

Sort Menu, by Target: [mnuCWSortTarget]

Reset the RelationChanges table sorting index to "TargetIndex"

Refi11 the change table [RefillRCTable]

Help Menu: Display the appropriate on-line help topics [Get_Help]

Controls: The following controls are defined for the ChangeWindow:

Elements Option [Optionl]: Display records from ElementChanges table Relations 0ption [0ption2]: Display records from Relationchanges table

Attributes Option [Option3]: Display records from AttributeChanges

ChangeGrid Table: Main table of the window to display change summaries

IEPrint Button: Print the current change table records

FirstPage Button [Mh3dButn1(0)]: Move to first Virtual Page

PreviousPage Button [Mh3dButn1(1)]: Move to the previous Virtual Page

NextPage Button [Mh3dButnl(2)]: Move to the next Virtual Page

LastPage Button [Mh3dButn1(3)]: Move to the last Virtual Page

PageLabel Text Box: Display current Virtual Page

Return Button [Command1]: Exit from the window

Form and Control Event Procedures: The event procedures below are used by the form and the controls listed above.

Sub Form Load - Triggered when the window is loaded

Resize the window, ChangeGrid, and position button and option controls

Set the correct option button to TRUE [Optionl, Option2, or 0ption3]

(Note that the Option buttons will fill the ChangeGrid) 
Sub Form Resize - Triggered when the window is resized by the user or software ScaTe the Changegrid to fit the window

Position the option buttons and control buttons

Sub Optionl Click - User selects the "Elements" view option

Enable and disable appropriate menus

Set sorting index

Fill the ChangeGrid table [RefillecTable]

Sub Option2_Click - User selects the "Relations" view option

Enable and disable appropriate menus

Set sorting index

Fill the Changegrid table [RefillRCTable]

Sub Option3 Click - User selects the "Attributes" view option

Enable and disable appropriate menus

Set sorting index

Fil1 the ChangeGrid table [Refil1ACTable]

Sub ChangeGrid_DblClick - User double-clicks on change history record Determine change record clicked

Display the appropriate window [ARecord, ERecord, or RRecord]

Sub IEPrint Click - User clicks the "Print" button

Determine the printing destination by displaying PrintDest window

Print the information to file or printer [FCRs or PCRs]

Sub PageLabel DblClick - User double-clicks the page label box

Get virtūal page number from user

Set new top virtual page

Refill window table [RefillACTable, RefillECTable, or RefillRCTable]

Sub FirstPage Click [Mh3dButn1(0)] - User clicks the "First Page" button Set top virtual page to 1

Refill window table [RefillaCTable, RefillECTable, or RefillRCTable]

Sub PreviousPage_Click [Mh3dButnl(1)] - User clicks "Previous Page" button Decrement top virtual page by 1

Refill window table [RefillACTable, RefillECTable, or RefillRCTable]

Sub NextPage Click [Mh3dButnl(2)] - User clicks the "Next Page" button Increment top virtual page by 1

Refill window table [RefillACTable, RefillECTable, or RefillRCTable]

Sub LastPage Click [Mh3dButnl(3)] - User clicks the "Last Page" button Set top virtual page to last page

Refil1 window table [RefillacTable, Refil1ECTable, or RefillRCTable]

Sub Command $1 \mathrm{Click}$ - User clicks the "Return" button

Unload and exit from the window 
WHC-SD-WM-SDR-012 Rev. 0

Additional Routines: The routines below are also defined in the Changewindow form.

Sub FACS - Prints all of the attribute changes to a file

For all records in the Attributechanges table

Print information to the print file [Filestring]

Sub FCRs - Prints all of the change information to a file

Open a new print output file

Call the appropriate printing routine [FACS, FECS, or FRCS]

Sub FECS - Prints all of the element changes to a file

For all records in the ElementChanges table

Print information to the print file [Filestring]

Sub FRCS - Print all of the relation changes to a file

For all records in the Relationchanges table

Print information to the print file [Filestring]

Sub NoCWButtons - Disables all of the virtual mode buttons

Disable and make invisible all of the virtual mode buttons

Sub PACS - Prints all of the attribute changes to the printer

For all records in the Attributechanges table

Print information to the printer [Printstring]

Sub PCRs - Prints all of the change information to the printer

Call the appropriate printing routine [PACS, PECS, or PRCS]

Sub PECS - Prints all of the element changes to the printer

For all records in the ElementChanges table Print information to the printer [PrintString]

Sub PRCS - Prints all of the relation changes to the printer

For all records in the Relationchanges table

Print information to the printer [Printstring]

Sub RefillacTable - Fills the change grid table with attribute change records Set the headers and columns of the Changegrid

For all Attributechange records to be displayed (all or virtual page) Add record to the ChangeGrid table

Set the virtual mode buttons [SetCWButtons]

Sub RefillECTable - Fills the change grid table with element change records

Set the headers and columns of the ChangeGrid

For all ElementChange records to be displayed (all or virtual page) Add record to the Changegrid table

Set the virtual mode buttons [SetCWButtons]

Sub RefillRCTable - Fills the change grid table with relation change records

Set the headers and columns of the Changegrid

For all Relationchange records to be displayed (all or virtual page) Add record to the Changegrid table

Set the virtual mode buttons [SetCWButtons] 
WHC-SD-WM-SDR-012 Rev. 0

Sub SetCWButtons - Sets the virtual mode buttons

Depending on the current virtual page, display the appropriate buttons

\subsubsection{AttributeChanges Record details window, ARecord (ARECORD.FRM)}

The ARecord window is used to display the detailed information for an AttributeChanges record. This window is displayed when an Attributechanges record is double-clicked in the ChangeGrid of the ChangeWindow. The user may change information in this window only to the Reason/Justification field. All other changes are discarded. The window is very similar in form and function to the ERecord and RRecord windows.

Menus: The ARecord form has the standard Edit and Help menus.

Menu Event Procedures: The Edit and Help menu event procedures are similar or identical to those of the MainWindow.

Controls: The following controls are used by the ARecord window:

IEFirst Button: Move to the first AttributeChange record

IEPrevious Button: Move to the previous AttributeChange record

IENext Button: Move to the next AttributeChange record

IELast Button: Move to the last AttributeChange record

Refresh Button [IEOIdViews]: Refresh the data

IEReturn Button: Exit from the window

Form and Control Event Procedures: The event procedures below are used by the ARecord form and the controls listed above.

Sub Form Load - Triggered when window is loaded

Go $\bar{t}_{0}$ the correct Attributechanges table record

Enable or disable the record navigation buttons

Fill in the data for the current record [FillAStuff]

Sub IEFirst Click - User clicks on the "First Record" button Update the current record [UpdateAChange]

Move to the first record in the AttributeChanges table

Fill in the data for the new record [FillAStuff]

Sub IEPrevious Click - User clicks on the "Previous Record" button Update the current record [UpdateAChange]

Move to the previous record in the AttributeChanges table

$F i 11$ in the data for the new record [Fil1AStuff]

Sub IENext Click - User clicks on the "Next Record" button Updat $\overline{\mathrm{e}}$ the current record [UpdateAChange]

Move to the next record in the Attributechanges table

Fill in the data for the new record [Fillastuff]

Sub IELast Click - User clicks on the "Last Record" button Update the current record [UpdateAChange]

Move to the last record in the AttributeChanges table

Fill in the data for the new record [Fillastuff] 
Sub IERefresh $\mathrm{Click}$ - User clicks on the "Refresh" button Refill the current data for the record [Fillastuff]

Sub IEReturn Click - User clicks on the "Return" button Update the current record [UpdateAChange]

Unload and exit from the window

Additional Routines: The following are additional routines that are defined within the ARecord window.

Sub Fillastuff - Fill in the window with the attribute change values Fill the text boxes with the details of the Attributechange record Enable and disable the record navigation buttons

Sub UpdateAChange - Save any change to the attribute change justification If Justification was changed, save the new justification value

\subsubsection{Elementchanges Record details window, ERecord (ERECORD.FRM)}

The ERecord window is used to display the detailed information for an ElementChange record. This window is displayed when an ElementChange record is double-clicked in the ChangeGrid of the ChangeWindow. The user may change information in this window only to the Reason/Justification field. All other changes are discarded. The window is very similar in form and function to the ARecord and RRecord windows.

Menus: The ERecord form has the standard Edit and Help menus.

Menu Event Procedures: The Edit and Help menu event procedures are similar or identical to those of the MainWindow.

Controls: The following controls are used by the ERecord window:

IEFirst Button: Move to the first ElementChange record

IEPrevious Button: Move to the previous ElementChange record

IENext Button: Move to the next Elementchange record

IELast Button: Move to the last ElementChange record

Refresh Button [IE01dViews]: Refresh the data

IEReturn Button: Exit from the window

Form and Control Event Procedures: The event procedures below are used by the ERecord form and the controls listed above.

Sub Form Load - Triggered when the window is loaded Go $\bar{t}_{0}$ the correct ElementChanges table record Enable or disable the record navigation buttons Fill in the data for the current record [Fi11EStuff]

Sub IEFirst Click - User clicks on the "First Record" button Update the current record [UpdateEChange]

Move to the first record in the ElementChanges table

Fill in the data for the new record [Fil1EStuff] 
Sub IEPrevious Click - User clicks on the "Previous Record" button Update the current record [UpdateEChange]

Move to the previous record in the ElementChanges table

Fill in the data for the new record [Fil1EStuff]

Sub IENext Click - User clicks on the "Next Record" button Update the current record [UpdateEChange]

Move to the next record in the ElementChanges table

Fill in the data for the new record [FillEStuff]

Sub IELast Click - User clicks on the "Last Record" button Update the current record [UpdateEChange]

Move to the last record in the ElementChanges table

Fill in the data for the new record [FillEStuff]

Sub IERefresh Click - User clicks on the "Refresh" button

Refill the current data for the record [Fillestuff]

Sub IEReturn Click - User clicks on the "Return" button Update the current record [UpdateEchange]

Unload and exit from the window

Additional Routines: The following are additional routines which are defined within the ERecord window.

Sub Fillestuff - Fills in the element change information

Fill the text boxes with the details of the ElementChange record

Enable and disable the record navigation buttons

Sub UpdateEChange - Updates any change to the element change justification If Justification was changed, save the new justification value

\subsubsection{Relationchanges Record details window, RRecord (RRECORD.FRM)}

The RRecord window is used to display the detailed information for a Relationchange record. This window is displayed when a Relationchange record is double-clicked in the ChangeGrid of the Changewindow. The user may change information in this window only to the Reason/Justification field. All other changes are discarded. The window is very similar in form and function to the ARecord and ERecord windows.

Menus: The RRecord form has the standard Edit and Help menus.

Menu Event Procedures: The Edit and Help menu event procedures are similar or identical to those of the MainWindow. 
Controls: The following controls are used by the RRecord window:

IEFirst Button: Move to the first RelationChange record

IEPrevious Button: Move to the previous Relationchange record

IENext Button: Move to the next Relationchange record

IELast Button: Move to the last RelationChange record

Refresh Button [IE0]dViews]: Refresh the data

IEReturn Button: Exit from the window

Form and Control Event Procedures: The event procedures below are used by the RRecord form and the controls listed above.

Sub Form Load - Triggered when the window is initially loaded

Go to the correct Relationchanges table record

Enable or disable the record navigation buttons

Fill in the data for the current record [FillRStuff]

Sub IEFirst_Click - User clicks on the "First Record" button

Update the current record [UpdateRChange]

Move to the first record in the Relationchanges table

Fill in the data for the new record [Fil1RStuff]

Sub IEPrevious Click - User clicks on the "Previous Record" button Update the current record [UpdateRChange]

Move to the previous record in the RelationChanges table

Fill in the data for the new record [FillRStuff]

Sub IENext Click - User clicks on the "Next Record" button Updat $\bar{e}$ the current record [UpdateRChange]

Move to the next record in the Relationchanges table

Fill in the data for the new record [FillRStuff]

Sub IELast Click - User clicks on the "Last Record" button Update the current record [UpdateRChange]

Move to the last record in the Relationchanges table

Fill in the data for the new record [FillRStuff]

Sub IERefresh Click - User clicks on the "Refresh" button Refill the current data for the record [FillRStuff]

Sub IEReturn Click - User clicks on the "Return" button Update the current record [UpdateRChange]

Unload and exit from the window

Additional Routines: The following are additional routines which are defined within the RRecord window.

Sub FillRStuff - Fill the window with the relation change information Fill the text boxes with the details of the Relationchange record Enable and disable the record navigation buttons

Sub UpdateRChange - Updates any change in the relation change justification If Justification was changed, save the new justification value 


\subsubsection{HistoryWindow (HISTORYW. FRM)}

The HistoryWindow is used to display all of the changes since a fixed baseline to a current element record being displayed in the ItemEdit window. To do this, the window displays information from the baseline and change history files located on the shared network server (currently $\backslash \backslash A P 001 \backslash S E-$ TOOLS). The window will allow the user to also display the differences between various versions of text attributes for the element through the selectcompare and Compare windows. The window consists of three tables for the element, attribute, and relation changes to the element, plus various buttons and menus. The window is resizable.

Menus: The HistoryWindow has the following menus:

Edit Menu: Same as the MainWindow Edit menu

Compare Menu: Does a comparison by displaying SelectCompare window

Help Menu: Similar to the MainWindow Help menu

Menu Event Procedures: The following are the event procedures for the menus Tisted above.

Edit Menu: Same as the MainWindow Edit menu.

Compare Menu Item: [mnuHCompare_Click] Get comparison to make and make it [GetCompare]

Help Menu: Displays the appropriate on-line help topic [Get_Help]

Controls: The following controls are found on the HistoryWindow:

EGrid Table: Displays element changes in history

AGrid Table: Displays attribute changes in history

RGrid Table: Displays relation changes in history

ReturnButton Button: Returns and exits from the window

Form and Control Event Procedures: The following are the events associated with the HistoryWindow form and the controls listed above.

Sub Form Load - Called when form is initially loaded

Initialize window size and grid columns/sizes

Fill in the data for the grids [FillHAGrid, FillHEGrid, FillHRGrid]

Sub Form Resize - Triggered when form is resized

Resize the tables to fit the window in width and height

Reposition labels and buttons

Sub AGrid Dblclick - User double-clicks on attribute change history record DispTay the attribute change information in the HARecord window

Sub EGrid DblClick - User double-clicks on element change history record DispTays the element change information in the HERecord window

Sub RGrid DblClick - User double-clicks on relation change history record DispTays the relation change information in the HRRecord window 
Sub ReturnButton Click - User clicks the "Return" button

Close the change history tables and database

Unload the Historywindow

Additional Routines: The following are additional routines which are defined within the HistoryWindow window.

Sub FillHAGrid - Fill the AGrid table with all attribute changes

For al1 changes in the AttributeChanges table for the current element Add attribute change history to AGrid

Sub FillHEGrid - Fill the EGrid table with all element changes

For all changes in the ElementChanges table for the current element Add element change history to EGrid

Sub FillHRGrid - Fill the RGrid table with all relation changes

For all changes in the Relationchanges table for the current element Add relation change history to RGrid

\subsubsection{History Attribute Record window (HARECORD.FRM)}

The HARecord window is used to display the detailed information for an Attribute Change History record. This window is displayed when an Attribute Change History record is double-clicked in the AGrid of the Historywindow. The user may not change any information in this window. The window is very similar in form and function to the HERecord and HRRecord windows.

Menus: The HARecord form has the standard Edit and Help menus.

Menu Event Procedures: The Edit and Help menu event procedures are similar or identical to those of the Mainwindow.

Controls: The following controls are used by the HARecord window:

IEFirst Button: Move to the first Attribute Change History record

IEPrevious Button: Move to the previous Attribute Change History record

IENext Button: Move to the next Attribute Change History record

IELast Button: Move to the last Attribute Change History record

IEReturn Button: Exit from the window

Form and Control Event Procedures: The event procedures below are used by the HARecord form and the controls listed above.

Sub Form Load - Triggered when window is loaded

FilT in the data for the current record [FillHAStuff]

Sub IEFirst $\mathrm{Click}$ - User clicks on the "First Record" button

Move to ${ }^{-}$the first record in the Attribute Change History table

Fill in the data for the new record [FillHAStuff]

Sub IEPrevious Click - User clicks on the "Previous Record" button Move to the previous record in the Attribute Change History table Fi1] in the data for the new record [FillHAStuff] 
Sub IENext Click - User clicks on the "Next Record" button

Move $\bar{F}_{0}$ the next record in the Attribute Change History table

Fill in the data for the new record [Fil1HAStuff]

Sub IELast Click - User clicks on the "Last Record" button

Move to the last record in the Attribute Change History table

Fill in the data for the new record [FillHAStuff]

Sub IEReturn Click - User clicks on the "Return" button

Unload and exit from the window

Additional Routines: The following are additional routines which are defined within the HARecord window.

Sub FillHAStuff - Fill in the window with the attribute change history values

Fill the text boxes with the Attribute Change History record

Enable and disable the record navigation buttons

\subsubsection{History Element Record window (HERECORD.FRM)}

The HERecord window is used to display the detailed information for an Element Change History record. This window is displayed when an Element Change History record is double-clicked in the EGrid of the Historywindow. The user may not change any information in this window. The window is very similar in form and function to the HARecord and HRRecord windows.

Menus: The HERecord form has the standard Edit and Help menus.

Menu Event Procedures: The Edit and Help menu event procedures are similar or identical to those of the MainWindow.

Controls: The following controls are used by the HERecord window:

IEFirst Button: Move to the first Element Change History record

IEPrevious Button: Move to the previous Element Change History record

IENext Button: Move to the next Element Change History record

IELast Button: Move to the last Element Change History record

IEReturn Button: Exit from the window

Form and Control Event Procedures: The event procedures below are used by the HERecord form and the controls listed above.

Sub Form Load - Triggered when window is loaded

FilT in the data for the current record [FillHEStuff]

Sub IEFirst Click - User clicks on the "First Record" button

Move to the first record in the Element Change History table

Fill in the data for the new record [FillHEStuff]

Sub IEPrevious CTick - User clicks on the "Previous Record" button Move to the previous record in the Element Change History table Fill in the data for the new record [FillHEStuff] 
Sub IENext Click - User clicks on the "Next Record" button

Move to the next record in the Element Change History table

Fill in the data for the new record [FillHEStuff]

Sub IELast Click - User clicks on the "Last Record" button

Move to the last record in the Element Change History table

Fill in the data for the new record [Fillitestuff]

Sub IEReturn Click - User clicks on the "Return" button

Unload and exit from the window

Additional Routines: The following are additional routines which are defined within the HERecord window.

Sub FillHEStuff - Fill in the window with the element change history values Fill the text boxes with the Element Change History record

Enable and disable the record navigation buttons

\subsubsection{History Relation Record window (HRRECORD. FRM)}

The HRRecord window is used to display the detailed information for a Relation Change History record. This window is displayed when a Relation Change History record is double-clicked in the RGrid of the Historywindow. The user may not change any information in this window. The window is very similar in form and function to the HARecord and HERecord windows.

Menus: The HRRecord form has the standard Edit and Help menus.

Menu Event Procedures: The Edit and Help menu event procedures are similar or identical to those of the Mainkindow.

Controls: The following controls are used by the HRRecord window:

IEFirst Button: Move to the first Relation Change History record

IEPrevious Button: Move to the previous Relation Change History record

IENext Button: Move to the next Relation Change History record

IELast Button: Move to the last Relation Change History record

IEReturn Button: Exit from the window

Form and Control Event Procedures: The event procedures below are used by the HRRecord form and the controls listed above.

Sub Form Load - Triggered when window is loaded

FilT in the data for the current record [FillHRStuff]

Sub IEFirst Click - User clicks on the "First Record" button

Move to the first record in the Relation Change History table

Fill in the data for the new record [FillHRStuff]

Sub IEPrevious Click - User clicks on the "Previous Record" button Move to the previous record in the Relation Change History table Fill in the data for the new record [FillHRStuff] 
Sub IENext Click - User clicks on the "Next Record" button Move $\bar{t}_{0}$ the next record in the Relation Change History table Fill in the data for the new record [FillHRStuff]

Sub IELast Click - User clicks on the "Last Record" button Move $\bar{t}_{0}$ the last record in the Relation Change History table Fill in the data for the new record [FillHRStuff]

Sub IEReturn_Click - User clicks on the "Return" button Unload and exit from the window

Additional Routines: The following are additional routines which are defined within the HRRecord window.

Sub FillHRStuff - Fill in the window with the attribute change history values Fill the text boxes with the Relation Change History record Enable and disable the record navigation buttons

\subsubsection{SelectCompare Window (SELECTCO.FRM)}

The Selectcompare window is used to identify which attribute to compare between which historical values, including the baseline and current values. The user selects an attribute, and two historical values to compare. The window will then display a "redline" comparison in the Compare window discussed below.

Menus: The SelectCompare window contains only the Help menu.

Menu Event Procedures: The Help menu event procedures are similar to the ones in the MainWindow.

Controls: The following are the controls on the SelectCompare window:

Attribute List List Box [AttrList]: Attribute for comparison

Listl List: First list of values for comparison

List2 List: Second list of values for comparison

CompareButton Button: Do the actual comparison

ReturnButton Button: Exit from the window

Form and Control Event Procedures: The following are the events associated with the Selectcompare window and the controls listed above.

Sub Form Load - Triggered when window is loaded Center the window on the screen

Sub AttrList Click - User selects attribute to be compared Determine attribute selected

Clear Listl and List?

Add all change history entries to Listl and List2 plus Current 
Sub CompareButton Click - User clicks on the "Compare" button

Determine chänge versions selected in Listl and List2

Find the values for the selected versions

Compare the values [CompareText]

Sub ReturnButton Click - User clicks the "Return" button

Unload the window and exit

\subsubsection{Compare Window (COMPARE.FRM)}

The Compare window is used to display the "redlined" results of a comparison between two historical values of an attribute for an element record. The window displays the old, new, and redlined values. The window is resizable.

Menus: The Compare window contains the standard Edit and Help menus.

Menu Event Procedures: The event procedures for the Edit and Help menus are the same as or similar to those of the MainWindow.

Controls: The following controls are found on the Compare window:

PrintButton Button: Prints the results

ReturnButton Button: Exits from the window

Form and Control Event Procedures: The following are the events associated with the Compare window and the controls listed above.

Sub Form Load - Triggered when window is loaded

Set the window size to the size of the screen

Sub Form Resize - Triggered when window is resized by the user or software Set the width and of the display boxes

Set the location of the button controls

Sub PrintButton Click - Prints the contents of the window to the printer Print the results to the printer [PrintDeltas]

Sub ReturnButton Click - Exits the window Unload and exit the window

\subsection{SUPPORT ROUTINE MODULE DESIGN DESCRIPTIONS}

These sections identify the Basic modules (.BAS files) which contain the support routines and functions for the software. Typically, the routines and functions are organized into groups of common functions. A short summary description of each routine is appended to the name. See Appendix A for the location of the routines and argument lists. 


\subsubsection{Global Variables and Constants (GLOBALS.BAS)}

The GLOBALS.BAS file identifies the constants and variables that are known globally to all forms and routines in the software. As such, there are no subroutines or functions defined, but definition for record structures, constants, and global variables. The outline below identifies the major ares of definition within this file.

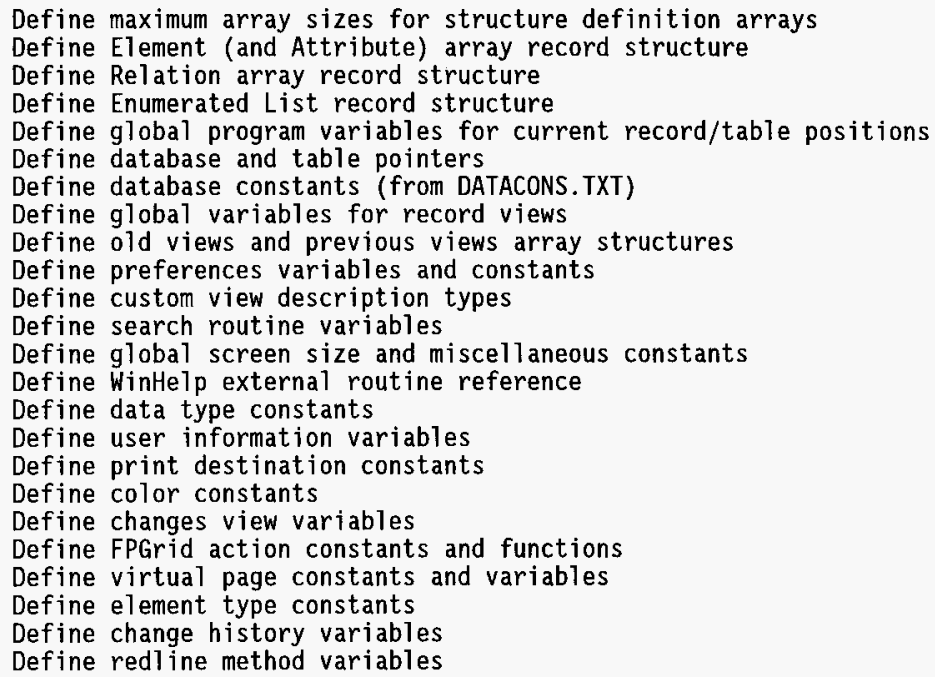

\subsubsection{Initialization Routines (INITIAL.BAS)}

The initialization routines are used generally to initialize various program parameters. The routines, and their functionality, are listed below.

Function GetDSetRelation - Get a string field from a dynaset

Get a string field value from a Dynaset, return "\# if \#NULL\#

Function GetDSI - Get an integer field from a dynaset

Get an integer field from a Dynaset, return 0 if \#NULL\#

Function GetElement - Get a string field from an element table

Returns a string field from an element table (superseded by GetString)

Function GetIR - Get an integer field from an element table

Return an integer field from an element table (superseded by GetInt)

Function GetRelation - Get a string field from a relation table

Returns a string field from a relation table (superseded by GetString) 
Sub InitProgram - Initialize the program

Initialize program constants (color, default settings, etc.)

Size, position, and initialize the MainWindow controls

Initialize the database structure [InitStructure]

Get the user's information [GetUserInfo]

Open initial database (query user if needed) [OpenTheDatabase]

Set the appropriate label nomenclature [SetLabels]

Set the element table indexes

Fill the main window grid [UpdateMainGrid]

Initialize other program variables

Sub InitRelGrid - Initialize the RelTable window's Relationgrid

Initialize the RelTable window controls and RelationGrid

Fill the Relationgrid [UpdateRelGrid]

Sub RefillMain - Refill the ElementGrid of the MainWindow

Clear the MainWindow ElementGrid table

Fill in the Number and Name fields for all rows in the ElementGrid

Sub SetRVButtons - Enable or disable the RelTable virtual mode buttons Set the virtual mode buttons for the RelTable window

Sub SetVButtons - Enable or disable the MainWindow virtual mode buttons Set the virtual mode buttons for the MainWindow

Sub UpdateMaingrid - Refill the MainWindow table

Determine size of grid (virtual mode or not) and initialize

Fill in the ElementGrid of the MainWindow form [RefillMain]

Add the deleted records to the ElementGrid

Update the virtual mode buttons [SetVButtons]

Sub UpdateRelGrid - Refill the RelTable window table

Determine the size of grid (virtual mode or not) and initialize

Fill in the Relationgrid of the RelTable form

Add the deleted records to the RelationGrid

Update the virtual mode buttons [SetRVButtons]

\subsubsection{Database Structure Initialization Routines (INITSTRU.BAS)}

The database structure initialization routines found in INITSTRU.BAS are used to initialize the database structure definition arrays, and open and close the database. The routines below are used within this file.

Sub Close4Export - Close an "Export to RMSB" file

Close Change tables and database for RMSB export file

Sub CloseTheDatabase - Close the currently open Browser database file Delete the temporary tables [DeleteTempTables]

close all tables and the database 
Sub Initstructure - Initialize \& define database structure definition arrays Define number of elements, relations, and base attributes

Initialize element names and nomenclature, and on line help topics

Initialize base attributes and relations for all elements

Set up additional attributes for each element type

Set up additional relations used by each element type

Initialize relation names and nomenclature, and on line help topics

Set up valid related element type 1 ists for all relations

Initialize all of the enumerated attribute lists

Sub Open4Export - Open an "Export to RMSB" file

Open change tables and database for exporting

Sub OpenTheDatabase - Open a Browser database file

Open database file and determine access level

Open element tables and set initial Index values

Open relation tables and set initial Index values

Open dataversion table

open change history tables

Create the temporary tables [CreateTempTables]

\subsubsection{Record Editing Routines (EDITING.BAS)}

The record editing routines in the file EDITING.BAS are used to do editing of a selected element record. These routines are typically called from the events in the ItemEdit window or one of the subordinate windows.

Function AddElement - Add a new element record

For all attributes, add the attribute value

For all attributes, save the attribute change history

Add a "Create" to the ElementChanges table [CreateElement]

Update main window if necessary [MGNewColor]

Sub Addoldview - add a view to the old views and previous view stacks Increment stack and place view on the stack

Sub AddRe1 - Add a relation link between elements

Check to make sure the relation doesn't previously exist

Add the relation and the inverse relation

Update the RelationChanges tables

Update attribute values for changed records (date time modified)

Function AddTheRelations - Add relations to elements from AddRelations window For all new element records to add the relation Add the relation link [AddRel]

Function CanSave - Determine if the record is valid to be saved (note, this will be modified significantly for Version 4.0 )

For all attributes, check the data types for errors

If a name, then make sure it is not blank

If it is a number, make sure it is valid [IsValidNumberField] 
Sub ChangedAttribute - Change a single attribute Look for previous AttributeChanges entry If previous, then modify previous, otherwise add new $A C$ record

Sub ColorRList - Fills in the RelationsGrid list in color For each related element entry

Determine color to display and change color

Sub CreateElement - Create a new element record change history entry Add "Create" record to ElementChanges table

Sub DeleteElement - Delete an element record from the database Delete all related Attributechanges records Delete all related Relationchanges records Remove all relation links [DeleteRelation] Remove all inverse relation links Delete the entry in the main window if necessary [MGDelete]

Find any ElementChanges records and modify, or add new one Delete any old or previous views to the element Delete the actual element record Add deleted "red" row to MainWindow table if necessary

Sub DeleteRelation - Delete a single relation link between elements Delete the selected relation Update the "color" of the source element Update the Relationchanges table with new relation delete record

Sub ElementRename - Rename an element record Rename all of the old views in the old views stack Change all of the relation table reference names Change all of the inverse relation name references Change all of the change table reference names Add rename change to ElementChanges history table Update the main window element grid [MGDelete, MGInsert, MGNewColor]

Sub ElementRenumber - Change the number attribute of an element record Renumber all of the old views in the old views stack Change all of the relation table reference numbers Change all of the inverse relation number references Update the main window element grid [MGDelete, MGInsert, MGNewCoTor]

Sub FileRecord - Output element record to a file open the output file and output header information Output the element attributes output the element relations close output file

Sub Filestring - Prints a string to the printer, with line breaks and spacing If string will fit on the line, just print it Otherwise, print the string breaking between words at 75 chars wide 
Sub FillElement - Fill in the attributes associated with an element record Initialize ItemEdit window and set navigation buttons [SetButtons]

Fill in values for all attributes (Loop around a Case statement)

Add the view to the views list [Add0ldview]

Set the attribute colors for any changed attributes

Fill in the relations grid [ColorRList, FillIERelGrid]

Sub FillFullRG - Fill as full the RelationsGrid of the ItemEdit window Initialize size and headers of the RelationsGrid Find all related elements and add to RelationsGrid

Add any deleted or removed elements to the RelationsGrid

Sub FilliERelGrid - Fill for one element type the RelationsGrid If fill all elements, then do so and exit [FillFullRG]

Initialize size and headers for the RelationsGrid of the ItemEdit window Find all related elements of the selected type and add to table Add any deleted or removed element links to the table

Sub InheritRels - Inherit relations from other elements

For all relations of a selected element add to the current element

Function InheritTheRelations - Inherit all relations from InheritRelations

For each element selected, inherit relations [InheritRels]

Function IsValidNumberfield - Determines if a Number attribute is valid

If number is null, then return True

Check each sequence for validity [IsValidSequence]

If all sequences valid, then return True, else return false

Function IsValidSequence - Determines if a character sequence is valid

If empty, then is not a valid sequence.

If all letters or all numbers, then return True, else return False

Sub Printall - Prints out the information for all element records for one type (note: Not currently used in the Browser)

For ail element records for a particular element table

Print header information

Print attributes

Print relations

Sub PrintRecord - Prints out the information for one element record

Print the header information

Print the attribute information

Print the relation information

Sub Printstring - Prints a string to the printer, with line breaks at 75 If string will fit on the line, just print it otherwise, print the string breaking between words at 75 chars wide 
Sub RemoveRelation - Removes a relation between elements

Remove any previous RelationChanges records

Remove the actual relationship link

Modify the attribute date and time on the source and target elements

Add the relation removal to the Relationchanges table

Delete the row from the RelationsGrid of the ItemEdit window

Add the relation row as a deleted row to the RelationsGrid

If necessary, update the main window [MGNewColor]

Sub SetButtons - Enables/Disables the record navigation buttons on ItemEdit

Enable or disable buttons according to current record position

Sub ShowElement - Displays an element record through the ItemEdit window

Verify and save current element, if any [CanSave, UpdateElement]

Fill in the relations 1 ist

For all attributes create (clone) a label

Depending on data type, calculate position of the label and attribute

Right justify all labels based on longest 1abel

Create and position all the attribute text, memo, and 1 ist boxes

Position the control buttons

Position the RelationGrid and REList list

Fill in the element data [FillElement]

Function UpdateElement - Update an element record

If read-only, then exit as True

If a new record, then add the new element record and exit

Determine which attributes have changed and save the change

Update the AttributeChanges (and other change tables) as necessary

\subsubsection{Search Routines (SEARCH.BAS)}

These routines are used to support the SearchWindow controls and functions. This includes the initialization of searches, doing the actual search, and displaying the results.

Sub AddToSearch - Adds a new clause to the search clause being built

Determine the attribute or relation chosen

Determine the related type and attribute (if any)

Determine which operation was selected

Determine the target value for the search

If search value type is invalid, say so and exit

Add any related element types to search clause as needed

Depending on the operation, build the search string accordingly

Update the HistoryGrid of the SearchWindow

If the operation was " then do the search [DoTheSearch]

Function CheckField - Determines if a value is correct for a given field type

For a string type, return True

If integer type, return value of IsNumeric function

For date or time type, return value of IsDate function

otherwise, return True 
Sub DoTheSearch - Executes the search of the database

Build the SQL search string

Create a QueryDef in the temporary database

Do the actual search (with a snapshot)

If no records found, display results and exit

Otherwise, fill the ResultsGrid with the name and numbers of records

Delete the QueryDef

\subsubsection{Data Export Routines (EXPORT.BAS)}

The file EXPORT.BAS contains the routines to export information out of the Browser in a variety of different formats.

Sub ASCIIChanges - Creates file of changes in ASCII (more readable than .RDT)

For all attribute and relation changes for an element

Print to file the attribute changes

Print to file the relation changes

Sub ASCIIHistory - Print out element history to ASCII text file

For all records in the Elementchanges table Output change information

Sub DBReport - Produces a record count summary report for the current database For a11 element tables print the record count number

For all relation tables print the record count number

For all change tables print the record count number

Sub ExportMatrix - Exports a Functions vs. Requirements matrix (tab delimited)

(Note: Functions are columns, Requirements are rows)

Get output file name from user

For all requirements (rows)

Clear related function array

Determine all related functions and put in function array

Output the row with " $X$ " for all related functions

Tell user when done

Sub ExportRMSB - Export a Browser format change file (Access format)

Get export filename from user

Copy all records from change tables to new export file

Sub InheritReqs - Was a temporary subroutine, currently not used.

Sub RDTChanges - Export the element changes in . RDT format

For all attribute and relation changes for an element

Print to file the attribute changes

Print to file the relation changes

Sub RDTHeader - Print the header information for an . RDT file

Print out the header line with RMSB version number

Sub RDTHistory - Print out element change history to . RDT file

For all elements in the ElementChanges table print a history entry 
Sub RDTPreface - Print the preface section of the . RDT file

Print out the preface information including date and user name

Sub ToASCIIText - Export text only report to an ASCII file

Check output file name

Output history information [ASCIIHistory]

Output change information [ASCIIChanges]

Sub ToSuperiorRDT - Export .RDT file as a subordinate Open the output. RDT file

Export header information [RDTHeader]

Export preface information [RDTPreface]

Export history information [RDTHistory]

Export changes information [RDTChanges]

\subsubsection{Edit Menu Routines (EDITMENU.BAS)}

The routines in EDITMENU.BAS provide the ability to interface with the Windows clipboard. This is done only for text, and not for graphics.

Generally speaking, for text boxes, the text is moved verbatim to and from the clipboard, and tables are moved to the clipboard as tab-delimited text.

Sub AppendToClipboard - Appends selected text to the Windows clipboard Get previous clipboard text

Determine type of ActiveForm and ActiveControl

Get text from Activecontrol according to type (text box, table, etc.)

Add new text to previous text and put on clipboard

Sub CopyToClipboard - Copies text to the clipboard Depending on ActiveControl type, get text

Put text on the clipboard

Sub CutToclipboard - Cuts text from control to the clipboard If not a text box, Beep and exit

cut text (delete) from active control and put on clipboard

Sub Pastefromclipboard - Paste text from the clipboard into control

If not a text box, beep and exit

0therwise replace selected text with clipboard text

Sub SelectA11Text - Select all text (or rows) in current control

Depending on the ActiveControl type, highlight/select all text items

Sub ShowClipboard - Display the contents of the clipboard

Determine clipboard contents

Display contents in frmExpand window 


\subsubsection{Miscellaneous Utility Routines (UTILITY.BAS)}

This file contains many of the generic routines which do not fit into one of the other categories, or are used in many different places in the software. These are typically small functions or routines which perform various

"utility" processes.

Sub CreateET - Creates an element type table in the database if does not exist Create the new table definition

Add all of the attributes to the table definition

Add the indexes to the table definition

Add the new table definition to the database

Sub CreateRT - Create a relation table in the database if it does not exist Create the new table definition

Add the table fields to the new table

Add the new indexes to the new table

Add the new table definition to the database

Sub CreateTempTables - Creates the temporary database tables (for searches) Create the temporary database file XXTEMPXX.MDB

Add the TwoTablequery results table

Add the Multiplequery results table

Add the ArchReport architecture report table

Sub DeleteTempTables - Deletes the temporary database tables

Close the temporary database

Delete the temporary database file

Function Even - Returns true if an integer is even (false if odd)

If $X \bmod 2=0$, return True else return False

Function FileExists - Determines if a file exists

Get attributes of file

If error, then return False, else return True

Sub FillCVAs - Fills in the Custom Value Attributes

For all of the base attributes, add the attribute names

For each of the additional attributes, add the attribute name and type

Sub Get Help - Shows an on-1ine help topic by number

CaTl the built-in WinHelp routine

Function GetDatestring - Returns a date attribute as a string or empty if null Get the date field from the selected table

If error, return ", else return the string value of the date

Function GetInt - Returns a short integer field from a table

Get the integer field from the selected table

If error, return zero, else return the value

Function GetLongInt - Returns a long integer field from a table

Get the long integer field from the selected table

If an error, return zero, else return the value 
Function GetNewFile - Get a new file name to open from the user Display a "file open" dialogue box to user

If canceled, return ", else, return the new file name

Function GetString - Get a string (or memo) field from a table

Get the string field value

If error, return " , else return the value

Function GetTheString - Another version of GetString to return a string field Get the string field value

If error, return ", else return the value

Function GetTimeString - Return a time string from a table attribute Get the time value from the field of the selected table

If error, return ", else return the time string

Function GetUserInfo - Get the user's information from their. INI file If the file does not exist, get user info [NewUser window]

Read all of the input parameters

If a custom view defined, load it [Fillcvas, SetCVAs]

Function GotoElementRecord - Finds/goes to a specified element record Depending on the current index, search by name or number and name If not found, then display a warning message and return False otherwise return True

Function GotoERQNoCase - Goes to an element record and ignores case changes Depending on the current index, search by name or number and name If not found, then return False

otherwise return True

Function GotoERQuiet - Goes to a specified element record without error msg Depending on the current index, search by name or number and name If not found, then return False

otherwise return True

Sub IRGColor - Set the color of a RelationsGrid row in the ItemEdit window For each column of the selected row

Make a picture of the text

Place picture in the image of the row

Sub LoadCustomView - Loads a custom view file Open the .CVF file

For all custom attributes, get the value

close file and exit

Sub MGColor - Sets the color of an ElementGrid row in the MainWindow

Select current row into block mode and change the color

Sub MGDelete - Deletes an ElementGrid row in the MainWindow

Find the appropriate grid row, if not found, exit

Select the row and delete 
Sub MGInsert - Insert an ElementGrid row in the MainWindow Determine new location for insert

Insert the new row

Sub MGNewColor - Change the color of an ElementGrid row in the MainWindow If new color is not blue or new color is blue and old was black then Change color of the row [MGColor]

Sub MoveServer - Tells the user the server has been moved (no longer used)

Function RepairTheDatabase - Repairs the current database file

Query user to continue

Close the current database file

Repair the current database file

If error, print warning message and return False

Reopen the current database and return True

Sub SavecurrentView - Save the current view parameters into a file Open the current view file

Output al1 of the custom view attribute settings

close the custom view file

Sub SaveCustomview - Save a current view into a file Open the current view file

output all of the custom view attribute settings

Close the custom view file

Sub SaveUserInfo - Save the user's preferences in the .INI file Open the. INI file

Write all parameters to the. INI file

Close the. INI file

Sub SetCVAs - Set the "Show" flag for all attribute fields

For all attributes in the database

Set the "Show" value for the attribute

Sub SetLabels - Sets all of the program labels based on chosen nomenclature For all element types, set the element label

For all relation types, set the relation labels

Sub SetSortorder - Sets the sorting order for an element table

Depending on the sort view, set the element table index

Sub ChnageDatabase - Opens a new database file

If new database file does not exist, exit

Close current database [CloseTheDatabase]

Unload all windows except main

Open the new database [OpenTheDatabase]

Set the labels [SetLabels]

Set indexes [SetSortorder]

Refill MainWindow grid [UpdateMaingrid]

Sub UpdateUpdate - Updates the Auto-Update program (No longer used) 


\subsubsection{Redlining routines (REDLINE.BAS)}

These routines are used for "redlining" text when doing a comparison between previous and current versions of text attributes. This window is displayed when two historical values are chosen from the Selectcompare window. The following are the routines which are defined in this file.

Sub CompareText - Compares and "redlines" two text values Determine the redline text [RedLine]

Fill the window with the input and reline information

Function EOSentence - Determines if word separators are an end of sentence If string in is empty, return False

If string contains a period, exclamation, or question mark, return True Otherwise return False

Function FindRenameAttr - Find new attribute value for a renamed element If no rename event found, return "-NOT FOUND-"

For each previous rename event seek the element value

If found, return the attribute value, otherwise return "-NOT FOUND-"

Function fPrintstring - Prints a string to the printer with indent, 75 chars If line will fit, print it and return

0therwise, keep splitting string at 75 chars or less (at spaces)

Print leftovers and return

Sub GetCompare - Initialize and display the SelectCompare window

Set SelectCompare window captions and values

For each memo or string attribute

Add the attribute name to the attribute list box

Display the SelectCompare window

Sub PrintDeltas - Prints the "redlined" comparison results to the printer

Print initial label identifying comparison

Print the old label and old text

Print the new label and new text

Print out the delta label and delta text by substituting the strikethrough and underline fonts for text markers

Function RedJunk - Returns the "redlined" difference between word delimiters

If old junk and new junk are the same, return junk

If old junk is empty, return inserted new junk

If new junk is empty, return deleted old junk

otherwise, determine differences and return redine 
Function RedLine - Returns the "redlined" difference between two strings If the two strings are equal, return them

Divide the two strings into "words" and "junk" (anything between words)

For each word in the new string

If matches current word in old string, increment both counters

If just changed case of words, delete old and insert new 0therwise determine closest matching words in both strings Delete old stuff and insert new stuff

Delete any old leftovers, and insert any new leftovers

Return the redline results

Sub ShowChangeHistory - Opens the change history file in HistoryWindow

If baseline file does not exist, print warning message

If change history file does not exist, print warning message and exit

open the change history database and display the Historywindow

\subsubsection{Additional search query routines (QUERY.BAS)}

These are routines to support the Multisearch and frmSinglequery windows. Originally written by Nancy Graves.

Function WhereSeg - Creates a SQL string for a search operation

Check for blanks (if exist, exit with null)

Check for valid value types

Create SQL string depending on search operation 
WHC-SD-WM-SDR-012 Rev. 0

This page intentionally left blank. 
WHC-SD-WM-SDR-012 Rev. 0

\subsection{REFERENCES}

WHC-CM-3-10, Software Practices, Revision 0, Appendix H, "System Design Description," Westinghouse Hanford Company, January 31, 1993.

WHC-SD-WM-CSCM-034, TWRS Systems Engineering Software Configuration Management Plan, Draft, dated September 1996. 
WHC-SD-WM-SDR-012 Rev. 0

This page intentionally left blank. 


\section{APPENDIX A}

\section{SUBROUTINE AND FUNCTION LIST}

The table below identifies all of the routines and functions used within the RMSB software. The routines are ordered first by the name of the routine, and then by the file name where the routine is found. For more information on each of the routines, refer to the appropriate file section previously mentioned, or the actual source code.

\begin{tabular}{|c|c|c|}
\hline File Name & Iype & Rout ine Name \\
\hline editing.bas & Function & AddE lement () As Integer \\
\hline editing bas & Sub & Addoldview (t As Integer, $n$ As string, num As string) \\
\hline editing.bas & Sub & $\begin{array}{l}\text { AddRel (WhichRel As Integer, SType As Integer, SName As String, SNumber As } \\
\text { String. RType As Integer, RName As String, RNumber As String) }\end{array}$ \\
\hline editing.bas & Function & AddTheRelations () As Integer \\
\hline search.bas & Sub & AddToSearch (TheOp As String) \\
\hline historyw. frm & Sub & AGrid Dblctick () \\
\hline edi tmenu. bas & Sub & AppendToCl ipboard () \\
\hline export.bas & Sub & ASCIIChanges () \\
\hline export.bas & Sub & ASCIIHistory () \\
\hline itemedit.frm & Sub & Attributelabel Dblclick (Index As Integer) \\
\hline itemedit.frm & sub & Attributelist $\mathrm{Click}$ (Index As Integer) \\
\hline itemedit.frm & Sub & AttributeMemo Change (Index As Integer) \\
\hline it emedit.frm & sub & Attributevalue Change (Index As Integer) \\
\hline searchwi.frm & Sub & AttrList_Click () \\
\hline selectco.frm & sub & AttrList $\mathrm{Click}(\mathrm{)}$ \\
\hline multsrch.frm & Sub & AttrList Click (Index As Integer) \\
\hline mul tsrch.frm & Sub & BPValueText Change (Index As Integer) \\
\hline multsrch.frm & sub & BRValueText Change (Index As Integer) \\
\hline customvi.frm & Sub & CancelButton $\mathrm{Click}()$ \\
\hline editing.bas & Function & Cansave () As Integer \\
\hline frmsary. frm & Sub & cbooperator Click (Index As Integer) \\
\hline utility.bas & Sub & ChangeDatabase (TFN As String) \\
\hline editing.bas & Sub & ChangedAttribute (EType As Integer, WATT As Integer, NewValue As string) \\
\hline changewi.frm & sub & ChangeGrid DblClick (Col As Long, Row As Long) \\
\hline search.bas & Function & Checkfield (FieldType As Integer, Fieldstring As String) As Integer \\
\hline multsrch.frm & Sub & Cleargrid () \\
\hline initstru.bas & sub & Close4Export () \\
\hline merge.bas & Sub & Closellergefile () \\
\hline initstru.bas & Sub & closeTheDatabase () \\
\hline
\end{tabular}




\section{WHC-SD-WM-SDR-012 Rev. 0}

\begin{tabular}{|c|c|c|}
\hline File Name & Type & Rout ine Name \\
\hline multsrch. frm & Sub & CmdAND Click () \\
\hline frmsqry.frm & Sub & cmdclose Click () \\
\hline frimsqry.frm & Sub & cmdEditRecord Click () \\
\hline frmsqry.frm & sub & cmdExecute $\mathrm{Click}(\mathrm{)}$ \\
\hline frmel sel.frm & Sub & cmoloK Click () \\
\hline frmexpan. frm & Sub & cmdoK click () \\
\hline listrela.frm & Sub & cmdoK_click () \\
\hline multsreh.frm & Sub & CMdOR $\mathrm{Cl}$ ick () \\
\hline frmsqry.frm & Sub & cmdReset click () \\
\hline multsrch.frm & sub & cmdReset click () \\
\hline multsrch.frm & Sub & cindReturn $\mathrm{Click}($ ) \\
\hline multsreh.frm & Sub & cmdsearch $\mathrm{click}(\mathrm{)}$ \\
\hline editing.bas & Sub & ColorRList (ET As Integer, EN As String) \\
\hline addrel at. $f$ rm & Sub & Command1 $\mathrm{Click}(\mathrm{)}$ \\
\hline changewi.frm & Sub & Cormand1_Click () \\
\hline frmelsel.frm & Sub & Command1 $\mathrm{click}($ ) \\
\hline getnewpa. $f r m$ & sub & Command1 $\mathrm{Cl}$ ick () \\
\hline getreaso.frm & sub & Command1 Click () \\
\hline inheritr.frm & Sub & Command1 $\mathrm{Cl}$ ick () \\
\hline mainwind.frm & Sub & Command1 $\mathrm{Click}()$ \\
\hline newuser.frm & sub & Command1 Click () \\
\hline oldviews.frm & Sub & Command1 $\mathrm{Click}()$ \\
\hline prefwind. frm & sub & Command1_ Click () \\
\hline printdes.frm & sub & Command1 $\mathrm{clifck}$ () \\
\hline searchwi.frm & sub & Command1 $\mathrm{Click}($ ) \\
\hline welcome. $f \mathrm{rm}$ & sub & Command1_Click () \\
\hline addrelat.frm & sub & Command2 Click () \\
\hline getnewpa. frm & sub & Command2 $\mathrm{Click}()$ \\
\hline getreaso.frm & Sub & Command2 $\mathrm{Click}(\mathrm{)}$ \\
\hline inheritr.frm & Sub & Command2 $\mathrm{Click}()$ \\
\hline mainwind. frm & Sub & Command2 $\mathrm{click}(\mathrm{)}$ \\
\hline newuser.frm & sub & Command2_Cl ick () \\
\hline oldviews.frm & Sub & Command2 $\mathrm{Click}($ ) \\
\hline prefwind.frm & sub & Commend2 $\mathrm{click}(\mathrm{)}$ \\
\hline printdes,frm & Sub & Command2 $\mathrm{Click}($ ) \\
\hline searchwi.frm & Sub & Command2 $\mathrm{Click}(\mathrm{)}$ \\
\hline wel come. f rm & Sub & Command2_click () \\
\hline
\end{tabular}




\begin{tabular}{|c|c|c|}
\hline File Name & Type & Rout ine Name \\
\hline mainwind. frm & Sub & Command3 Click () \\
\hline printdes. frm & Sub & Command3 $\mathrm{Cl}$ ick () \\
\hline searchwi.frm & Sub & Command3_ $\mathrm{Cl}$ ick () \\
\hline mainwind.frm & sub & Command4 $\mathrm{Click}()$ \\
\hline searchwi.frm & Sub & Cormand4 $\mathrm{Click}()$ \\
\hline mainwind. frm & Sub & Command5 $\mathrm{Cl}$ ick () \\
\hline searchwi.frm & sub & Cormand5_Click () \\
\hline searchwi .frm & Sub & Command6 $\mathrm{Click}()$ \\
\hline selectco.frm & Sub & CompareButton Click () \\
\hline redl ine.bas & sub & $\begin{array}{l}\text { CompareText coldText As String, oldtitle As String, Newtext As String, NewTitle } \\
\text { As String, CompareLabel As String) }\end{array}$ \\
\hline editmenu. bas & Sub & CopyTocl ipboard () \\
\hline editing.bas & Sub & CreateE lement (EType As Integer, newname As String) \\
\hline utility-bas & Sub & CreateET (i As integer) \\
\hline utility.bas & Sub & CreateRT (i As Integer) \\
\hline utility,bas & sub & CreateTempiables () \\
\hline editmenu. bas & sub & CutTocl i pboard () \\
\hline merge.bas & sub & DandRE lements (AttributeChanges As Table) \\
\hline export.bas & Sub & DBReport (TFN As String) \\
\hline merge.bas & Sub & DelACs (StartRec As Integer, EndRec As Integer, Queryuser As Integer) \\
\hline merge.bas & sub & $\begin{array}{l}\text { DelECs (StartRec As Integer, EndRec As Integer, Queryuser As Integer, } \\
\text { AttributeChanges AS Table) }\end{array}$ \\
\hline editing.bas & Sub & DeleteElenent (EType As Integer, TheName As string, TheNum As string) \\
\hline editing.bas & Sub & $\begin{array}{l}\text { DeleteRelation (TheRel As Integer, SType As Integer, SName As String, SNumber } \\
\text { As String, RType As Integer, RName As String, RNumber As String) }\end{array}$ \\
\hline utility.bas & Sub & DeleteTempTables () \\
\hline merge.bas & sub & DelRCs (StartRec As Integer, EndRec As Integer, QueryUser As Integer) \\
\hline cust omvi.frm & sub & Displaycvas () \\
\hline search.bas & sub & DotheSearch () \\
\hline historyw.frm & Sub & EGrid Dblclick () \\
\hline mainwind. frm & sub & ElementGrid Dblclick (Col As Long, Row As Long) \\
\hline mainwind.frm & Sub & ElementList $\mathrm{Cl}$ ick () \\
\hline editing.bas & Sub & ElementRename (EType As Integer, OldName As string, OldNumber As string) \\
\hline editing.bas & Sub & ElementRenumber (EType As Integer, oldNumber As String) \\
\hline multsrch.frm & Sub & Element Type $\mathrm{Cl}$ ick (Index As Integer) \\
\hline redl ine.bas & Function & EoSentence (Instring As string) As integer \\
\hline utility.bas & Function & Even ( $x$ As Integer) As Integer \\
\hline export . bas & Sub & ExportMatrix (TheFileName As String) \\
\hline export.bas & Sub & ExportRMSB () \\
\hline
\end{tabular}


WHC-SD-WM-SDR-012 Rev. 0

\begin{tabular}{|c|c|c|}
\hline File Name & Type & Rout ine Name \\
\hline changewi. frm & Sub & FACS (\rangle \\
\hline changewi.frm & Sub & FCRs (NewFileName As string) \\
\hline changewi . frm & Sub & FECS () \\
\hline utility.bas & Function & FileExists (TheFile As String) As Integer \\
\hline editing.bas & Sub & FileRecord (ThefileName As String) \\
\hline editing bas & Sub & Filestring (thestring As String, $\mathrm{fl}$ As Integer, Indent As Integer) \\
\hline arecord. frm & Sub & Fillastuff () \\
\hline utility.bas & Sub & Fill CVAs () \\
\hline editing.bas & Sub & FillElement (EType As Integer) \\
\hline erecord. frm & Sub & Fillestuff () \\
\hline editing.bas & Sub & FillFul IRG (EType As Integer) \\
\hline mul tsrch.frm & Sub & Fillgrid 0 \\
\hline historyw.frm & Sub & FillHAGrid () \\
\hline harecord. frm & Sub & Fil.tHAStuff () \\
\hline historyw. frm & Sub & FillHEGrid () \\
\hline herecord. frm & Sub & FillHESTUff () \\
\hline historyw, frm & Sub & Fill HRGrid () \\
\hline hrrecord, frm & Sub & Fillthestuff () \\
\hline editing.bas & Sub & Fill IEReLGrid (EType As Integer) \\
\hline rrecord.frm & Sub & Fillastuff 0 \\
\hline merge.bas & Function & FindElement (EType As Integer, EName As string) As string \\
\hline redl ine.bas & Function & FindRenameattr ( $T$ As Table, WA As Integer) As String \\
\hline listrela.frm & Sub & Form Activate () \\
\hline mainwind. frm & Sub & Form LinkError (LinkErr As Integer) \\
\hline mainwind. frm & Sub & Form LinkExecute (CmdStr As String, Cancel As Integer) \\
\hline addrelat.frm & Sub & Form Load () \\
\hline arecord.frm & Sub & Form Load () \\
\hline changewi, frm & Sub & Form Load () \\
\hline compare. frm & Sub & Form Load () \\
\hline custornvi f frm & Sub & Form Load () \\
\hline erecord.frm & Sub & Form_Load () \\
\hline frmelsel.frm & Sub & Form Load () \\
\hline frmexpan. frm & Sub & Form Load () \\
\hline frmsgry. frm & Sub & Form Load () \\
\hline getnewpa. frm & Sub & Form Load () \\
\hline getreaso, frm & Sub & Form Load () \\
\hline harecord.frm & Sub & Form_Load () \\
\hline
\end{tabular}




\begin{tabular}{|c|c|c|}
\hline File Name & Type & Rout ine Name \\
\hline herecord.frm & Sub & Form Load () \\
\hline historyw. frm & Sub & Form Load () \\
\hline hrrecord.frm & Sub & Form Load () \\
\hline inheritr.frm & Sub & Form Load $(2)$ \\
\hline itemedit.frm & Sub & Form Load () \\
\hline listrela.frm & Sub & Form Load () \\
\hline mainwind. frm & Sub & Form Load () \\
\hline mul tsrch. frm & Sub & Form Load () \\
\hline newuser. frm & Sub & Form Load () \\
\hline oldviews.frm & Sub & Form Load () \\
\hline prefwind.frm & Sub & Form Load () \\
\hline printdes, frm & Sub & Form Load 0 \\
\hline rel table.frm & Sub & Form Load () \\
\hline rrecord.frm & Sub & Form Load () \\
\hline searchwi.frm & Sub & Form Load () \\
\hline selectco.frm & Sub & Form Load () \\
\hline wel come.frm & Sub & Form Load () \\
\hline addrelat.frm & Sub & Form Resize () \\
\hline changewi.frm & Sub & Form Resize () \\
\hline compare, frm & sub & Form Resize () \\
\hline historyw.frm & Sub & Form Resize () \\
\hline inheritr.frm & Sub & Form Resize () \\
\hline mainwind. frm & Sub & Form_Resize () \\
\hline oldviews.frm & Sub & Form Resize () \\
\hline reltable.frm & Sub & Form Resize () \\
\hline searchwi.frm & Sub & Form Resize () \\
\hline redt ine.bas & Function & fPrintstring (thestring As string, fI As Integer, Indent As Integer) As Integer \\
\hline changewi.frm & Sub & $\operatorname{FRCS}()$ \\
\hline redline.bas & Sub & GetCompare () \\
\hline utility.bas & Function & GetDatestring (TheTable As Table, thefield As String) As String \\
\hline initial.bas & Function & GetDSetRelation (TheDS As Dynaset, FieldName As String) As String \\
\hline initial.bas & Function & GetDSI (TheDS As Dynaset, fieldName As String) As Integer \\
\hline initial bas & Function & GetE lement (theindex As Integer, fieldName As String) As String \\
\hline utility.bas & Function & GetInt (TheTable As Table, thefield As String) As Integer \\
\hline initial .bas & Function & GetIR (theindex As Integer, FieldNane As String) As Integer \\
\hline utility.bas & Function & GetLongInt (TheTable As Table, thefield As String) As Long \\
\hline utility.bas & Function & GetNewFile (Title As String, Ext As String, Filter As String) As String \\
\hline
\end{tabular}




\begin{tabular}{|c|c|c|}
\hline File Name & Iype & Rout ine Name \\
\hline initial bas & Function & GetRelation (theindex As Integer, FieldName As String) As String \\
\hline utility.bas & Function & GetString (TheTable As Table, thefield As String) As String \\
\hline ut ility.bas & Function & $\begin{array}{l}\text { GetTheString (TheTable As Table, EType As Integer, TheAttribute As Integer) As } \\
\text { String }\end{array}$ \\
\hline utility.bas & Function & GetTimeString (TheTable As Table, thefield As String) As String \\
\hline utility.bas & Sub & GetUserInfo () \\
\hline utility.bas & Sub & Get Help (HelpTopic As Long) \\
\hline utility.bas & Function & GotoE lementRecord (t As Integer, $n$ As string, num As String) As Integer \\
\hline utility.bas & Function & GotoERQNoCase ( $t$ As Integer, $n$ As String, num As string) \\
\hline utility.bas & Function & GotoERQuiet (t As integer, $n$ As String, num As string) As Integer \\
\hline frmsqry. frm & Sub & Grid1 Dblclick () \\
\hline itemedit.frm & Sub & HelpIEMenus $\mathrm{Cl}$ ick () \\
\hline merge.bas & Sub & IDCAttributes (AttributeChanges As Table) \\
\hline merge.bas & Sub & IDCCreate () \\
\hline merge.bas & Sub & IDCDelete () \\
\hline merge. bas & Sub & IDCE L ements () \\
\hline merge.bas & Sub & IDConflicts (AttributeChanges As Table) \\
\hline merge.bas & Sub & IDCRelations () \\
\hline merge.bas & Sub & IDCRename () \\
\hline itemedit.frm & Sub & IECancel $\mathrm{Click}()$ \\
\hline arecord.frm & sub & IEFirst click () \\
\hline erecord.frm & Sub & IEFirst Click () \\
\hline harecord.frm & Sub & IEFirst Click () \\
\hline herecord.frm & Sub & IEFirst Click () \\
\hline hrrecord. frin & sub & IEFirst Click () \\
\hline itemedit.frm & Sub & IEFirst click () \\
\hline rrecord.frm & sub & IEFirst_click () \\
\hline arecord.frin & Sub & IELast Click () \\
\hline erecord.frm & Sub & IELast Click () \\
\hline harecord.frm & sub & IELast Click () \\
\hline herecord.frm & sub & IELast click () \\
\hline hrrecord.frm & Sub & IELast Click () \\
\hline itemedit.frm & Sub & IELast_click () \\
\hline rrecord.frm & sub & IELast $\mathrm{Cl}$ ick () \\
\hline arecord.frm & Sub & IENext Click () \\
\hline erecord. frm & Sub & IENext Click () \\
\hline harecord.frm & Sub & IENext Click () \\
\hline herecord.frm & Sub & IENext_click () \\
\hline
\end{tabular}


WHC-SD-WM-SDR-012 Rev. 0

\begin{tabular}{|c|c|c|}
\hline File Name & Type & Rout ine Name \\
\hline hrrecord.frm & Sub & IEHext click () \\
\hline itemedit.frm & Sub & IENext_click () \\
\hline rrecord. frm & sub & IENext Click () \\
\hline arecord.frm & sub & IEOLOViews Click () \\
\hline erecord. frm & Sub & IEOLViews Click () \\
\hline itemedit.frm & Sub. & IEOLdViews Click () \\
\hline rrecord.frm & Sub & IEOldViews_click () \\
\hline arecord.frm & Sub & IEPrevious $\mathrm{Cl}$ ick () \\
\hline erecord.frm & Sub & IEPrevious $\mathrm{Click}()$ \\
\hline harecord.frm & sub & IEPrevious click () \\
\hline herecord.frm & sub & IEPrevious click () \\
\hline hrrecord.frm & Sub & IEPrevious Click () \\
\hline itemedit, frm & sub & IEPrevious Click () \\
\hline rrecord.frm & Sub & IEPrevious Click () \\
\hline changewi.frm & Sub & IEPrint_click () \\
\hline itemedit.frm & Sub & IEPrint click () \\
\hline mainwind. frm & sub & IEPrint Click () \\
\hline oldviews.frm & sub & IEPrint_click () \\
\hline reltable.frm & Sub & IEPrint $\mathrm{Cl}$ ick () \\
\hline searchwi.frm & sub & IEPrint Click () \\
\hline arecord.frm & Sub & IEReturn click () \\
\hline erecord.frm & sub & IEReturn $\mathrm{Click}($ ) \\
\hline harecord.frm & sub & IEReturn_click () \\
\hline herecord.trm & Sub & IEReturn Click () \\
\hline hrrecord.frm & Sub & IEReturn Click 0 \\
\hline it temedit. frm & Sub & 1EReturn $\mathrm{Click}()$ \\
\hline rrecord.frm & Sub & IEReturn Click () \\
\hline merge.bas & Sub & IncAttributes () \\
\hline merge.bas & Sub & IncConflicts () \\
\hline merge.bas & Sub & IncEl ements () \\
\hline merge.bas & sub & Incorpattributes (Queryuser As Integer) \\
\hline merge.bas & Sub & IncorpCreate (Queryuser As Integer) \\
\hline merge.bas & sub & IncorpDelete (Queryuser As Integer) \\
\hline merge.bas & sub & IncorpE lements (QueryUser As Integer) \\
\hline merge.bas & Sub & IncorporateAll (Queryuser As Integer) \\
\hline merge.bas & sub & IncorpRelations (Queryuser As Integer) \\
\hline merge. bas & sub & IncorpRename (Queryuser As Integer) \\
\hline
\end{tabular}


WHC-SD-WM-SDR-012 Rev. 0

\begin{tabular}{|c|c|c|}
\hline File Name & Type & Rout ine Name \\
\hline merge.bas & Sub & IncRelations () \\
\hline editing.bas & Sub & $\begin{array}{l}\text { InheritRels (WhichRel As Integer, SType As Integer, SName As String, SNumber As } \\
\text { String, RType As Integer, RName As String, RHumber As String, TheRType As } \\
\text { Integer) }\end{array}$ \\
\hline export.bas & Sub & InheritRegs (ThefileName As String) \\
\hline editing.bas & Function & InheritTheRelations () \\
\hline initial.bas & Sub & InitProgram () \\
\hline initial.bas & sub & InitRelGrid () \\
\hline initstru.bas & sub & Initstructure () \\
\hline utility.bas & Sub & IRGColor (TheRow As Integer, Thecolor As Long) \\
\hline editing.bas & Function & IsVal idNumberfield (TheNumber As String) As Integer \\
\hline editing_bas & Function & IsValidSequence (seq As String) As Integer \\
\hline arecord. $\mathrm{frm}$ & Sub & JField Change () \\
\hline erecord.frm & Sub & JField Change () \\
\hline harecord.frm & Sub & Jfield Change () \\
\hline herecord.frm & Sub & JField Change () \\
\hline hrrecord.frm & sub & JField Change () \\
\hline rrecord.frm & Sub & JField Change () \\
\hline itemedit.frm & Sub & List1 $\mathrm{click}()$ \\
\hline prefwind. frm & Sub & List 1 Click 0$)$ \\
\hline frmsqry. frm & sub & LoadControls () \\
\hline utility.bas & Sub & LoadCustonView (TFN As String) \\
\hline multsrch.frm & Sub & MakeSQL () \\
\hline frmsqry. $f \mathrm{rm}$ & Function & MakeSQL () As Integer \\
\hline merge.bas & Sub & MergeAppendAll () \\
\hline merge.bas & Sub & MergeAttributes (Query As Integer, AttributeChanges As Table) \\
\hline merge.bas & Sub & MergeCreate (Query As Integer, AttributeChanges As Table) \\
\hline merge.bas & sub & Mergebelete (Query As Integer, Attributechanges As Table) \\
\hline merge.bas & Sub & MergeE (ements (Query As Integer) \\
\hline merge.bas & Sub & Mergefiles (Query As Integer, Attributechanges As Table) \\
\hline merge.bas & Sub & MergeRelations (Query As Integer) \\
\hline merge.bas & Sub & MergeRename (Query As Integer, Attributechanges As Table) \\
\hline utility.bas & sub & MGColor (TheRow As Integer, TheColor As Long) \\
\hline utility.bas & Sub & MGDelete (whichname As string) \\
\hline utility.bas & Sub & MGInsert (whichname As String, whichnum As String) \\
\hline utility.bas & Sub & MGNewColor (whichname As String, NewColor As Long) \\
\hline addrelat.frm & Sub & Mh3dButn1 click (Index As Integer) \\
\hline changewi.frm & Sub & Mh3dButn1_cl ick (Index As Integer) \\
\hline
\end{tabular}




\begin{tabular}{|c|c|c|}
\hline File Name & Type & Rout ine Name \\
\hline inheritr.frm & Sub & Mh3obutn1 Click (Index As integer) \\
\hline mainwind. frm & Sub & Mh3dButn1_Click (Index As Integer) \\
\hline rel table, frm & sub & Mh3dButn1 Click (Index As Integer) \\
\hline maimwind.frm & Sub & mnuabout $\mathrm{Click}()$ \\
\hline arecord. frm & Sub & mnuACCon $\mathrm{Cl}$ ick () \\
\hline arecord.frm & sub & muACCopy $\mathrm{Cl}$ ick () \\
\hline arecord. frm & Sub & mnuACCut $\mathrm{Click}()$ \\
\hline arecord. frm & Sub & mnUACDC $\mathrm{Cl}$ ick () \\
\hline arecord.frm & sub & mnUACEM $\mathrm{Cl}$ ick () \\
\hline arecord. frm & Sub & mnUACGI $\mathrm{Click}()$ \\
\hline arecord. frm & Sub & mnuACPaste $\mathrm{Cl}$ ick () \\
\hline arecord. frm & Sub & mnuACRAppend $\mathrm{Cl}$ ick () \\
\hline arecord. frm & Sub & mnuACSAll $\mathrm{click}()$ \\
\hline arecord, frm & Sub & mnUACTW $\mathrm{Click}()$ \\
\hline arecord. frm & Sub & minuACUH $\mathbf{c}$ (ick () \\
\hline itenedit.frm & Sub & minuaddRe lation_Click () \\
\hline itemedit.frm & Sub & mnuArchRept Click () \\
\hline changewi f frm & sub & minucccon $c($ ick () \\
\hline changewi f frm & Sub & mnuCCDAppend Click () \\
\hline changewi.frm & Sub & mnUCCEM Click () \\
\hline changewi , frm & sub & mnuCCGI Click () \\
\hline changewi f frm & sub & mnucctH $\mathrm{c}$. ick () \\
\hline changewi f frm & sub & minuccuH_click () \\
\hline reltable,frm & sub & mnuCGI Click () \\
\hline historyw. frm & sub & mnuCHWAppend $\mathrm{C}$ i ick () \\
\hline historyw.frm & Sub & mnuCHWCopy Click () \\
\hline historyw.frm & sub & mnuchWCut_click () \\
\hline historyw.frm & Sub & mnUCHWC $\mathrm{Cl}$ ick () \\
\hline historyw.frm & Sub & mnuCHWHelpCon click () \\
\hline historyw.frm & Sub & mnuCHWHelpGi $\mathrm{Click}($ ) \\
\hline historyw.frm & Sub & mnuCHWHelpTw_Click () \\
\hline historyw. frm & Sub & mnucHWHelpUH Click () \\
\hline historyw. frm & Sub & mnuCHWPaste $\mathrm{Click}()$ \\
\hline historyw. frm & Sub & mnUCHWSA $C l$ ick () \\
\hline itemedit. frm & Sub & mnucopy Click () \\
\hline customvi.frm & sub & mnucveon click () \\
\hline custormvi.frm & Sub & mnUCVGInfo_click () \\
\hline
\end{tabular}




\begin{tabular}{|c|c|c|}
\hline File Name & Type & Rout ine Name \\
\hline customvi.frm & Sub & mnucvopen $\mathrm{Click}()$ \\
\hline customvi.frm & Sub & mnucvSaveAs Click () \\
\hline customvi.frm & Sub & mnucvsave click () \\
\hline customvi.frm & Sub & mnuCVuHelp Click 0 \\
\hline changewi.frm & Sub & mnuCWCopy Click () \\
\hline changewi. frm & Sub & mnuCwCut $\mathrm{Click}()$ \\
\hline changewi.frm & Sub & mnuCWDC_Click () \\
\hline changewi f. frm & Sub & mnuCWPaste $\mathrm{Click}()$ \\
\hline changewi.frm & Sub & mnuCWSelectall Click () \\
\hline changewi.frm & Sub & mnuCwSortchange $\mathrm{Click}($ ) \\
\hline changewi . frm & Sub & mnuCWSortE lement_Cl ick () \\
\hline changewi,frm & sub & mnuCWSortTarget Click () \\
\hline changewi.frm & Sub & mnuCWSortTime $\mathrm{Click}()$ \\
\hline mainwind. frm & Sub & mnuDataversion $\mathrm{Click}()$ \\
\hline mainwind. frm & sub & mnuBBReport_click () \\
\hline itemedit.frm & Sub & mnuDeleteRelation_Click () \\
\hline erecord.frm & Sub & mnuEccon $\mathrm{Cl}$ ick () \\
\hline erecord.frm & Sub & mnuEcCopy $\mathrm{Cl}$ ick () \\
\hline erecord. frm & Sub & minuECCut_Click () \\
\hline erecord.frm & Sub & mnuECDC $\mathrm{Click} \cup$ \\
\hline erecord, frm & Sub & manuECEM Click () \\
\hline erecord. frm & Sub & manuECGI $C$ lick () \\
\hline erecord.frm & Sub & mnuECPaste_click () \\
\hline erecord. frm & Sub & mnuECRAppend Click () \\
\hline erecord.frm & Sub & mnuECSA $\mid$ Click () \\
\hline erecord. frm & Sub & mnuECTH $C($ ick () \\
\hline erecord. frm & Sub & mnuECUH Click () \\
\hline frmsgry. frm & sub & monuExit $\mathrm{Click}()$ \\
\hline mainwind. frm & Sub & mnuExit click () \\
\hline mul tsrch.frm & Sub & mnuExit click () \\
\hline mainwind. frm & Sub & mnuExportRMSB_Click () \\
\hline mainwind. frm & Sub & mnuFECtoText click () \\
\hline mainwind. frm & Sub & mnuF i leopen $\mathrm{Cl}$ ick () \\
\hline mainwind. frm & sub & mnuFRMatrix $\mathrm{Cl}$ ick () \\
\hline frmsqry. frm & Sub & MnUFSOCSET Click () \\
\hline harecord. frm & sub & mnuHACCon $\mathrm{Click}()$ \\
\hline harecord. frm & sub & mnuHACCOpy_Cl ick () \\
\hline
\end{tabular}




\begin{tabular}{|c|c|c|}
\hline File Name & Type & Rout ine Name \\
\hline harecord. frm & Sub & muHACCut $\mathrm{Click}(\mathrm{)})$ \\
\hline harecord.frm & Sub & muHACDC $\mathrm{Cl}$ ick () \\
\hline harecord.frm & sub & InUHACEM Click () \\
\hline harecord.frm & Sub & mnUHACGI Click 0 \\
\hline harecord.frm & Sub & mnuHACPaste click () \\
\hline harecord.frm & Sub & mnUHACRAppend Click () \\
\hline harecord. frm & Sub & mnUHACSALI_Click () \\
\hline harecord. frm & sub & mnUHACTW Click () \\
\hline harecord.frm & Sub & mกUHACUH CLick () \\
\hline historyw. frm & Sub & mnuHCompare $\mathrm{Cl}$ ick () \\
\hline herecord.frm & Sub & mnuHECCOn_Click () \\
\hline herecord.frm & Sub & mnuHECCopy $\mathrm{Cl}$ ick $\mathrm{O}$ \\
\hline herecord. frm & Sub & mnuHECCut $\mathrm{Cl}$ ick () \\
\hline herecord. frm & Sub & mnuHECOC Click () \\
\hline herecord. frm & sub & mnUHECEM_Click () \\
\hline herecord. frm & Sub & mnuHECGI $C$ Lick () \\
\hline herecord. frm & Sub & mnUHECPaste $\mathrm{Cl}$ ick () \\
\hline herecord. frm & Sub & mnuHECRAPpend_click () \\
\hline herecord.frm & Sub & mRUHECSALL Click () \\
\hline herecord. frm & Sub & mnUHECTW $\mathrm{Cl}$ ick 0 \\
\hline herecord, frm & Sub & IMUHECUH $\mathrm{Click}($ ) \\
\hline mainwind. frm & Sub & monuHelpMwButtons $\mathrm{click}()$ \\
\hline mainwind. frm & Sub & mnuHelpMwMenus_click () \\
\hline addrelat. frm & Sub & mulHelp Click () \\
\hline inheritr.frm & Sub & mnuHelp Click () \\
\hline hrrecord. frm & Sub & mnuHRCCon Click () \\
\hline hrrecord.frm & Sub & mnuHRCCopy Click () \\
\hline hrrecord.frm & Sub & mnuHRCCut Click () \\
\hline hrrecord.frm & Sub & muHRCDC Click () \\
\hline hrrecord.frm & Sub & mnUHRCEM Click () \\
\hline hrrecord.frm & Sub & mnUHRCGI Click () \\
\hline hrrecord.frm & Sub & mnuHRCPaste $\mathrm{Click}()$ \\
\hline hrrecord. frm & sub & mnuHRCRAppend CLick () \\
\hline hrrecord.frm & Sub & mnuHRCSAll Click () \\
\hline hrrecord.frm & Sub & mnUHRCTW Click () \\
\hline hrrecord. frm & Sub & InUHRCUH Click () \\
\hline itemedit.frm & Sub & mnulEAppend_Click () \\
\hline
\end{tabular}




\begin{tabular}{|c|c|c|}
\hline File Name & Type & Rout ine Name \\
\hline itemedit.frm & sub & mulEAtts $\mathrm{Click}()$ \\
\hline itemedit.frm & Sub & mnuIEB_Click () \\
\hline itemedit.frm & Sub & mulECopy $\mathrm{Click}($ ) \\
\hline itemedit. frm & Sub & mnulECut $\mathrm{Click}()$ \\
\hline i temedit.frm & Sub & AnulEC Click () \\
\hline itemedit.frm & Sub & mnuIEDC Click () \\
\hline itemedit.frm & Sub & mnuIEE Is_click () \\
\hline itemedit.frm & Sub & mUIEEM_Click () \\
\hline itemedit. frm & Sub & mnulEGI $\mathrm{Click}()$ \\
\hline itemedit.frm & Sub & muIEGoto $\mathrm{Click}($ ) \\
\hline itemedit. frm & Sub & mnulEInherit click 0 \\
\hline itemedit.frm & Sub & mnuIEMQuery $\mathrm{Click}(\mathrm{O}$ \\
\hline itemedit.frm & Sub & mnulEOL dViews_click () \\
\hline itemedit. frm & Sub & mulepaste $c l i c k()$ \\
\hline itemedit.frm & Sub & mnuIEQsingle click () \\
\hline itemedit.frm & sub & mnuIERels CLick () \\
\hline itemedit.frm & Sub & mnulESelectAll click () \\
\hline itemedit.frm & Sub & mnulEThisAttr click () \\
\hline i temedit. frm & Sub & mnuIETh is $\mathrm{Click}()$ \\
\hline itemedit.frm & Sub & mnuIETwoIable $\mathrm{Cl}$ ick () \\
\hline itemedit.frm & Sub & moulEIW Click () \\
\hline itemedit. frm & Sub & mnUIEUH click () \\
\hline itemedit.frm & Sub & mnulEVCH_Click () \\
\hline mainwind. frm & Sub & minumainCopy $\mathrm{Click}(\mathrm{)}$ \\
\hline mainwind. frm & Sub & mnumaincut $\mathrm{Click}()$ \\
\hline mainwind. frm & Sub & mnuMaindBstruct_Click () \\
\hline mainwind. $\mathrm{frm}$ & Sub & mnuMaindelete $\mathrm{Click}()$ \\
\hline mainwind, frm & sub & mnuMainErase $\mathrm{Click}(\mathrm{)}$ \\
\hline mainwind, frm & Sub & mnumainNew Click () \\
\hline mainwind. frm & Sub & mnuMainPaste $\mathrm{Click}()$ \\
\hline mainwind. frm & sub & mnumainselectall_click () \\
\hline mainwind. frm & Sub & mutmainThisElement click () \\
\hline mainwind. frm & Sub & mnumwappend Click () \\
\hline mainwind. $\mathrm{frm}$ & sub & muntwl click () \\
\hline mainwind. frm & Sub & mnuMwDC Click () \\
\hline mainwind. frm & sub & mnUMWEM Click 0 \\
\hline mainwind. frm & Sub & mnuHWG_Click () \\
\hline
\end{tabular}




\begin{tabular}{|c|c|c|}
\hline File Name & Type & Rout ine Name \\
\hline mainwind. $f r m$ & sub & mnuMLMw Click () \\
\hline mainwind. frm & sub & mnuMHeMul tiple $\mathrm{click}($ ) \\
\hline mainwind. frm & Sub & mnuwwQs ingle $\mathrm{Cl}$ ick () \\
\hline mainwind. frm & Sub & muNWWH Click () \\
\hline mainwind. frm & Sub & mnuMWVH $\mathrm{Click}($ ) \\
\hline itemedit.frm & Sub & mnuNewRecord Click () \\
\hline oldviews. frm & sub & mnuovAppend_Click () \\
\hline oldviews.frm & Sub & mnuovcopy $\mathrm{Click}($ ) \\
\hline oldviews.frm & Sub & mnuovcut $\mathrm{Click}()$ \\
\hline oldviews. frm & Sub & mnuove $\mathrm{Cl}$ ick () \\
\hline oldviews.frm & Sub & mRUOVEM Cl ick () \\
\hline oldviews.frm & Sub & mnuovg Click () \\
\hline oldviews.frm & Sub & mnuovPaste Click () \\
\hline oldviews.frm & Sub & mnuovselectall click () \\
\hline oldviews.frm & Sub & mnuOVTW_Click () \\
\hline oldviews. frm & Sub & mnuovuH $\mathrm{click}()$ \\
\hline oldviews. frm & sub & mnuPEVDC $\mathrm{Cl}$ ick () \\
\hline prefwind. frm & Sub & mnuPrefHelp Click () \\
\hline mainwind. frm & Sub & mnuPrefs $\mathrm{Cl}$ ick () \\
\hline frmsqry. frm & Sub & mnuprint $\mathrm{Click}()$ \\
\hline multsreh.frm & Sub & mnuPrint $\mathrm{Cl}$ ick () \\
\hline frmsqry.frm & Sub & mnuOCopy Click () \\
\hline frmsqry.frm & Sub & mnuQCut Click () \\
\hline frmsqry. frm & Sub & mnuac Click () \\
\hline frmsqry.frm & Sub & mnLGEM Click () \\
\hline frmsqry.frm & Sub & mnUOG I Cl ick () \\
\hline frmsqry. frm & Sub & mnuaPaste click () \\
\hline frmsgry. frm & Sub & mugselectall click () \\
\hline frmsqry. frm & Sub & mnuesw Click () \\
\hline frmsqry.frm & Sub & mnuquH $\mathrm{Cl}$ ick () \\
\hline rrecord.frm & Sub & mnuRCCon $\mathbf{c}$ lick () \\
\hline rrecord.frm & Sub & mnuRCCopy Click () \\
\hline rrecord. frm & Sub & mnuRCCut $\mathrm{Click}($ ) \\
\hline rrecord, frm & sub & mmuRCOC $\mathrm{Cl}$ ick () \\
\hline rrecord.frm & sub & mnURCEM $\mathrm{Click}($ ) \\
\hline rrecord. $\mathrm{frm}$ & sub & mnURCGI Click 0 \\
\hline rrecord.frm & Sub & mnuRCPaste_click () \\
\hline
\end{tabular}




\begin{tabular}{|c|c|c|}
\hline File Name & Type & Rout ine Name \\
\hline rrecord. frm & Sub & mnuRCRAppend Click () \\
\hline rrecord. frm & sub & mmuRCSAll click () \\
\hline rrecord.frm & sub & mnURCTH Click () \\
\hline rrecord. frm & Sub & mnURCUH $\mathrm{Click}()$ \\
\hline itemedit.frm & sub & mnuRecordbelete click () ' \\
\hline compare frm & Sub & mnuRedAppend $\mathrm{Cl}$ ick $\mathrm{O}$ ) \\
\hline compare. frm & Sub & mnuRedCopy Click () \\
\hline compare. frm & sub & muRedCut $\mathrm{click}($ ) \\
\hline compare. frm & Sub & mnuRedoc $\mathrm{Click}()$ \\
\hline compare.frm & Sub & mnuRedHelpCon cl ick () \\
\hline compare. frm & Sub & mnuRedHelpGI click () \\
\hline compare. $\mathrm{frm}$ & sub. & mnuRedHelpTW Click () \\
\hline compare.frm & Sub & muRedHelpuH $\mathrm{Cl}$ ick () \\
\hline compare. frm & sub & mnuRedPaste $\mathrm{Cl}$ ick () \\
\hline compare. frm & Sub & mnURedSA_Click () \\
\hline reltable.frm & Sub & mnuRelRels $C l$ ick () \\
\hline rel table. frm & sub & minuReLThisRel Click () \\
\hline mainwind. frm & sub & mnuRepair Click () \\
\hline reltable.frm & sub & mnuRTAppend $\mathrm{Cl}$ ick () \\
\hline rel table.frm & sub & mmuRTCopy $C$ lick () \\
\hline reltable.frm & Sub & mnuRTCut_Clíck () \\
\hline rel table.frm & Sub & manuRTC Click () \\
\hline reltable.frm & sub & mnURTDC Click () \\
\hline reltable.frm & Sub & mnURTEM Click () \\
\hline rel table. frm & sub & mnuRTPaste $\mathrm{Click}()$ \\
\hline reltable.frm & sub & mnuRTSelectall Click () \\
\hline reltable.frm & Sub & mnuRTTW Click () \\
\hline reltable.frm & Sub & mnURTUH $\mathrm{Click} 0$ \\
\hline selectco.frm & sub & mnuscwhelpCon $\mathrm{Cl}$ ick () \\
\hline selectco. frm & sub & mnuSCWHelpgI Click () \\
\hline selectco.frm & Sub & mnuSCWHelpTw Click () \\
\hline selectco.frm & Sub & mnuSCwHelpuH $\mathrm{Cl}$ ick () \\
\hline searchwi.frm & Sub & mnuSearch Copy $\mathrm{Cl}$ ick () \\
\hline mainwind. $f r m$ & sub & mnushowchanges click () \\
\hline frmsgry. $f r m$ & sub & nnuShowSQL Click () \\
\hline multsrch.frm & sub & mnuShowSQL Click () \\
\hline frmsqry.frm & sub & mnusortName_Click () \\
\hline
\end{tabular}




\begin{tabular}{|c|c|c|}
\hline File Name & Type & Rout ine Name \\
\hline multsrch.frm & Sub & mnusortName $\mathrm{click}()$ \\
\hline frmsqry.frm & Sub & mnusor tNumber Click () \\
\hline multsrch.frm & sub & mnusor tNumber click () \\
\hline multsrch.frm & sub & mnusortRBywame $\mathrm{Click}()$ \\
\hline multsrch.frm & Sub & mnuSortRBy Nunber $\mathrm{Cl}$ ick () \\
\hline printdes.frm & Sub & mnuSPDH $\mathrm{Cl}$ ick () \\
\hline frmsgry. frm & sub & mnuSQWDC Click () \\
\hline frmsqry. frm & Sub & mnUSQYAppend CLick () \\
\hline searchwi.frm & Sub & mnusrchoc $\mathrm{Cl}$ ick () \\
\hline mainwind. frm & Sub & muSupRDT Click () \\
\hline searchwi.frm & Sub & mnuSWAppend Click () \\
\hline multsrch.frm & sub & mnuSwCopy $\mathrm{Click}(\mathrm{)}$ \\
\hline searchwi.frm & Sub & mnuSWCopy Click () \\
\hline searchwi.frm & Sub & mnUSWCST CLICK () \\
\hline multsrch.frm & Sub & mnuSwCut_Click () \\
\hline searchwi.frm & Sub & mnuSWCut Click () \\
\hline multsrch.frm & Sub & mnusWC $\mathrm{Click}()$ \\
\hline searchwi.frm & Sub & mnuswC_click () \\
\hline multsrch.frm & Sub & mnUSWEM Click () \\
\hline searchwi.frm & Sub & MnUSWEM $C($ ick () \\
\hline multsrch.frm & sub & muSWGI Click () \\
\hline searchwi.frm & Sub & mnuSWGI $\mathrm{Click}()$ \\
\hline multsrch.frm & Sub & mruSWPaste_Click () \\
\hline searchwi.frm & Sub & mnuswPaste $\mathrm{click}()$ \\
\hline searchwi.frm. & Sub & mnusuSB $\mathrm{Click}()$ \\
\hline searchwi.frm & Sub & mnuswsC $\mathrm{Click}()$ \\
\hline multsrch, frm & Sub & mnususelectAl l click () \\
\hline searchwi.frm & Sub & mnuSWSelectall Click $S)$ \\
\hline searchwi.frm & Sub & mnuSWSW $\mathrm{Cl}$ ick () \\
\hline multsrch.frm & Sub & mnuswuH $\mathrm{Click}()$ \\
\hline searchwi.frm & Sub & monUSWUH $\mathrm{Cl}$ ick () \\
\hline multsrch.frm & Sub & mnuTTSAppend Click () \\
\hline multsrch.frm & sub & mnuTTSDC Click () \\
\hline multsrch.frm & sub & munUTSH $\mathrm{Cl}$ ick () \\
\hline mainwind.frm & Sub & muTworable click () \\
\hline newuser.frm & sub & mnusserHelo $\mathrm{Cl}$ ick () \\
\hline utility.bas & Sub & Moveserver () \\
\hline
\end{tabular}


WHC-SD-WM-SDR-012 Rev. 0

\begin{tabular}{|c|c|c|}
\hline File Name & Type & Rout ine Name \\
\hline printdes.frm & Sub & NewFil leName Change () \\
\hline changewi.frm & sub & NoCWBut tons () \\
\hline customvi.frm & sub & OKButton Click () \\
\hline reltable,frm & sub & oldviews (lick () \\
\hline initstru.bas & Sub & Open4Export (TFN As String) \\
\hline merge.bas & Sub & Opentog2 0 \\
\hline merge.bas & Sub & OpenLogfile () \\
\hline merge.bas & Sub & OpenMergefile () \\
\hline initstru.bas & Sub & OpentheDatabase (TFN As String) \\
\hline multsrch.frm & Sub & OpList Click (Index As Integer) \\
\hline changewi.frm & Sub & Option1 $\mathrm{Click}()$ \\
\hline changewi.frm & sub & Option2 $\mathrm{click}()$ \\
\hline changewi.frm & Sub & Option3 Click () \\
\hline changewi.frm & sub & PACS () \\
\hline addrelat. frm & Sub & Pagelabel_Click () \\
\hline inheritr.frm & sub & PageLabel Click () \\
\hline changewi.frm & Sub & Pagelabel Dblclick () \\
\hline mainwind. $\mathrm{frm}$ & sub & Pagelsbel Dblclick () \\
\hline reltable.frm & sub & Pagelabel Dblctick () \\
\hline editmenu.bas & sub & Pastefromclipboard () \\
\hline changewi.frm & Sub & PCRs () \\
\hline changewi.frm & sub & PECS () \\
\hline changewi.frm & Sub & PRCS () \\
\hline editing.bas & sub & Printall () \\
\hline compare. $\mathrm{frm}$ & Sub & PrintButton $\mathrm{Click}()$ \\
\hline redt ine.bas & sub & PrintDel tas (Thetext As String) \\
\hline editing.bas & Sub & PrintRecord () \\
\hline editing -bas & Sub & Printstring (thestring As String, $f($ As Integer, Indent As Integer) \\
\hline mainwind. frm & Sub & QuitButton Click () \\
\hline reltable,frm & sub & QuitB Click () \\
\hline export.bas & Sub & RDTChanges () \\
\hline export_bas & Sub & RDTHeader () \\
\hline export.bas & Sub & RDTHistory () \\
\hline export.bas & Sub & RDTPreface () \\
\hline redl ine.bas & Function & Reddunk (Mewdunk As string, OldJunk As string) As string \\
\hline redl ine. bas & Function & Redl ine (oldstring As string, Newstring As String) As String \\
\hline changewi.frm & Sub & Refillactable () \\
\hline
\end{tabular}




\begin{tabular}{|c|c|c|}
\hline File Name & Type & Rout ine Name \\
\hline changewi.frm & Sub & Refillectable () \\
\hline initial.bas & sub & Refill Main (NumRows As Integer) \\
\hline changewi.frm & Sub & RefillRCTable () \\
\hline rel table.frm & Sub & RelationGrid DblClick (Col As Long, Row As Long) \\
\hline reltable.frm & Sub & Relationlist click () \\
\hline mainwind. frm & Sub & RelationsB Click () \\
\hline itemedit.frm & Sub & RelationsGrid DblClick () 'Col As Long, Row As Long) \\
\hline searchwi.frm & Sub & RelattrList click () \\
\hline itemedit.frm & Sub & REList $\mathrm{Cl}$ ick () \\
\hline searchwi.frm & Sub & RelTypelist Click () \\
\hline editing.bas & sub & $\begin{array}{l}\text { RemoveRelation (TheRel As Integer, SType As Integer, SName As string, SNumber } \\
\text { As String, RType As Integer, RName As String, RNumber As String) }\end{array}$ \\
\hline utility.bas & Function & RepairTheDatabase () As integer \\
\hline multsrch.frm & Sub & ResultsGrid Dblclick () \\
\hline searchwi.frm & Sub & ResultsGrid Dblclick () \\
\hline compare. frm & sub & ReturnButton click () \\
\hline historyw.frm & Sub & ReturnButton Click () \\
\hline selectco.frm & Sub & ReturnButton $\mathrm{Cl}$ ick () \\
\hline historyw.frm & Sub & RGrid Dblclick () \\
\hline addre lat.frm & Sub & RList Click () \\
\hline inheritr. frm & sub & RList Click () \\
\hline utility.bas & Sub & SaveCurrentView (IFN As string) \\
\hline utility.bas & sub & SaveCustomview (TFN As string) \\
\hline utility.bas & Sub & SaveuserInfo () \\
\hline mainwind. frm & Sub & SearchButton_click () \\
\hline edi tmenu, bas & Sub & Selectalltext () \\
\hline addrelat.frm & Sub & SetAvButtons () \\
\hline inheritr.frm & sub & SetAVButtons () \\
\hline editing bas & Sub & SetButtons (EType As Integer) \\
\hline utility.bas & Sub & SetcVAs () \\
\hline changewi f frm & sub & SetCWButtons () \\
\hline utility.bas & Sub & SetLabels (Newiview As Integer) \\
\hline initial.bas & Sub & SetRVButtons () \\
\hline ut il ity.bas & sub & SetSortorder (i As Integer, Neworder As Integer) \\
\hline initial,bas & sub & Setviuttons () \\
\hline redl ine.bas & Sub & ShowChangeH istory (TheType As Integer, TheName As String) \\
\hline editmenu.bas & Sub & ShowCl ipboard () \\
\hline editing.bas & Sub & ShowE lement (EType As Integer, ename As String, ENumber As String) \\
\hline
\end{tabular}


WHC-SD-WM-SDR-012 Rev. 0

\begin{tabular}{|c|c|c|}
\hline File Name & Type & Rout ine Name \\
\hline mainwind. frm & Sub & SortButton $\mathrm{Click}()$ \\
\hline reltable.frm & Sub & SortB Click () \\
\hline export.bas & Sub & ToAscIllext (ThefileName As string) \\
\hline export,bas & Sub & ToSuperiorRDT (ThefileName As String) \\
\hline arecord. frm & Sub & UpdateAChange () \\
\hline erecord. frm & Sub & UpdateEChange () \\
\hline editing.bas & Function & UpdateE lement () As Integer \\
\hline initial.bas & Sub & UpdateMaingrīd () \\
\hline rrecord.frm & Sub & UpdateRChange () \\
\hline initial.bas & Sub & UpdateRelGrid () \\
\hline ut ility.bas & Sub & UpdateUpdate () \\
\hline mul tsrch. frm & Sub & ValueL ist Change (Index As Integer) \\
\hline mul tsrch.frm & Sub & Valuelext Change (Index As Integer) \\
\hline oldviews.frm & Sub & ViewsGrid DblClick () \\
\hline prefwind.frm & Sub & VMode Click () \\
\hline frmsqry. frm & Sub & vsMovechild Change () \\
\hline addrelat. from & sub & VTList $\mathrm{Click}(\mathrm{V}$ \\
\hline inheritr.frm & Sub & VIL ist Click () \\
\hline query.bas & Function & $\begin{array}{l}\text { Whereseg (AttrName As String, AttrType As Integer, OpType As Integer, value As } \\
\text { String, valuel As string) As String }\end{array}$ \\
\hline
\end{tabular}




\section{DISTRIBUTION SHEET}

\begin{tabular}{|c|c|c|c|c|c|c|}
\hline \multirow{2}{*}{$\begin{array}{l}\text { To } \\
\text { Distribution }\end{array}$} & \multirow{2}{*}{\multicolumn{4}{|c|}{$\begin{array}{l}\text { From } \\
\text { SE Risk Management \& Modeling }\end{array}$}} & \multicolumn{2}{|l|}{ Page 1 of 1} \\
\hline & & & & & \multicolumn{2}{|c|}{ Date $9 / 26 / 96$} \\
\hline \multicolumn{5}{|l|}{ Project Title/Work Order } & \multicolumn{2}{|c|}{ EDT No. $618229=$} \\
\hline \multicolumn{5}{|c|}{$\begin{array}{l}\text { Requirements Management System Browser Software Design } \\
\text { Description }\end{array}$} & ECN No. & \\
\hline Name & & MSIN & $\begin{array}{c}\text { Text } \\
\text { With All } \\
\text { Attach. }\end{array}$ & Text Only & $\begin{array}{l}\text { Attach./ } \\
\text { Appendix } \\
\text { Only }\end{array}$ & $\begin{array}{l}\text { EDT/ECN } \\
\text { Only }\end{array}$ \\
\hline
\end{tabular}

WHC

N. G. Awadalla

D. O. Frank

B. C. Gneiting

N. J. Graves

L. G. Peck

D. F. Salsman

J. M. Vann

$\underline{\mathrm{RL}}$

D. J. Francis

other

Central Files (Original)

Log Book (D. D. Frank)

$\begin{array}{ll}H 6-35 & X \\ H 6-35 & X \\ H 6-35 & X \\ H 6-35 & X \\ H 6-35 & X \\ H 6-35 & X \\ H 6-33 & X\end{array}$

K6-51 $X$

A3-88

H6-35 $x$
$x$
$x$
$x$
$x$
$x$
$x$

$x$

$x$
$x$ 